\title{
COHOMOLOGY CLASSES ASSOCIATED TO ANOMALIES
}

\author{
GREGORY LAMBROS PETERSON
}

\begin{abstract}
One of the proposed settings for the description of anomalies in the setting of gauge field theories is a local bicomplex associated to a principal fiber bundle $G \rightarrow P \rightarrow M$. It is a bigraded algebra with two differentials which is invariantly defined, i.e. independent of local coordinates of $M$. I denote it by $S_{M}^{\bullet, \bullet}$. Briefly, $S_{M}^{p, q}$ consists of local $p$-multilinear operators from the gauge algebra into $q$-forms on $M$ which depend on a connection $A$ in a local manner; local means that the operators decrease supports. The gauge algebra is the Lie algebra of the gauge group, which consists of diffeomorphisms of $P$ that respect the action of $G$ and cover the identity diffeomorphism of $M$. In this setting, the anomalies are described as integrals over $M$ whose integrands can be shown to be representatives of total cohomology classes in $H^{1}\left(S_{M}^{\bullet, \bullet}\right)$.

The main reason for restricting to a local bicomplex is due to Peetre's theorem. It states that local operators are differential operators over open sets $U \subset M$. This property is both mathematically natural and required by physical considerations.

This paper explores the computation of the total cohomology of the local bicomplex by beginning with the coordinate description of the differential operators and then determining which of these differential operators can be used to construct invariantly defined objects. What is accomplished is the description of the differential operators which are invariant under the action of the local diffeomorphisms of $\mathbb{R}^{n}$ and the computation of their total cohomology over open sets $U \subset M$. The main result is that

$$
H_{d}^{\bullet}\left(\left(S_{U}^{\bullet, \bullet}\right)^{\operatorname{Diff}_{\mathrm{loc}}\left(\mathbf{R}^{n}\right)}\right) \simeq H_{d}^{\bullet}\left(W(\mathfrak{g})_{\left[\frac{n}{2}\right]}\right),
$$

where $\left(S_{U}^{\bullet, \bullet}\right)^{\text {Diffloc }}\left(\mathbf{R}^{n}\right)$ denotes the invariant differential operators over the open set $U$ and $W(\mathfrak{g})_{\left[\frac{n}{2}\right]}$ is the Weil algebra of $\mathfrak{g}$, the Lie algebra of $G$ truncated at $\left[\frac{n}{2}\right]$, the greatest integer less than or equal to half the dimension of $M$. This shows that the cohomology groups over open sets are nonzero only in the range $n \leq q \leq n+r$ where $r$ is the dimension of the Lie algebra $\mathfrak{g}$, and in this range they are all finite dimensional. This result is globalized in the special case that the associated fiber bundle ad $^{*} P$ is trivializable.
\end{abstract}

\section{INTRODUCTION}

1.1. A brief review of the relevant differential geometry. Let $P$ be a principal fiber bundle, $G \rightarrow P \stackrel{\pi}{\rightarrow} M$, where $G$ is a compact, connected Lie group of dimension $r$ and $M$ is a compact, oriented, Riemannian manifold of dimension $n$. Thus $G$ is both the fiber and the structure group of $P$. Furthermore

Received by the editors September 10, 1990 and, in revised form, September 23, 1991.

1991 Mathematics Subject Classification. Primary 53A55, 53B50, 53C07, 53C80, 58H99, 81T13.

Key words and phrases. Gauge group, gauge algebra, cohomology, invariants, Weil algebra, anomalies. 
the action of $G$ on $P$, (which is on the right) is smooth, free, transitive, and effective. Hence, for each $g \in G$ there is an element of $\operatorname{Diff}(P)$, the group of diffeomorphisms of $P$, denoted by $R_{g}$, where $R_{g}(p)=p g$. The Lie algebra of $G$ will be denoted by $\mathfrak{g}$.

The gauge group $\mathscr{G}$ is the group of vertical automorphisms of $P$. These are diffeomorphisms $\Theta$ of $P$, which cover the identity map of $M$ and respect the action of $G$ on $P$, i.e. $\pi(\Theta(p))=\pi(p)$ and $\Theta(p g)=\Theta(p) g$. It is shown in [1] that $\mathscr{G}$ is isomorphic to $\Gamma(\operatorname{Ad} P)$, the $C^{\infty}$ sections of the associated fiber bundle Ad $P$. Recall that Ad $P$ is the set of equivalence classes of $P \times G$, where $(p, g) \sim\left(p^{\prime}, g^{\prime}\right)$, if there exists an $h \in G$ such that $(p, g)=$ $\left(p^{\prime} h, h^{-1} g^{\prime} h\right)$. Note that the fibers of Ad $P$ are diffeomorphic to $G$.

The gauge algebra $\mathbf{g}$ is the "Lie algebra" of the gauge group $\mathscr{G}$. The gauge algebra can be identified as either the vertical, $G$-invariant vector fields on $P$, (which will be denoted by $\mathscr{X}(P)_{V}^{G}$, where $\mathscr{X}(P)$ denotes all vector fields on $P)$, or as $\Gamma($ ad $P)$, the $C^{\infty}$ sections of the associated fiber bundle ad $P$. On the one hand, a vector field $X$ is called vertical if $X_{p} \in \operatorname{ker} \pi_{*}: T_{p} P \rightarrow T_{\pi(p)} M$ for all $p \in P$ and $X$ is called $G$-invariant $R_{g^{*}}\left(X_{p}\right)=X_{p g}$ for all $g \in G$. On the other hand, ad $P$ is the set of equivalence classes of $P \times \mathfrak{g}$ where the representation of $G$ in $\operatorname{Aut}(\mathfrak{g})$ is by the adjoint action. Thus $(p, x)$ is equivalent to $\left(p^{\prime}, x^{\prime}\right)$ if there is a $g \in G$ such that $(p, x)=\left(p^{\prime} g, \operatorname{Ad}\left(g^{-1}\right) x^{\prime}\right)$ where $x$ and $x^{\prime}$ are in $\mathfrak{g}$. Note that the fibers of ad $P$ are isomorphic to the Lie algebra $\mathfrak{g}$. Consequently $\mathbf{g}$ has a Lie bracket $[$, ] defined by the pointwise use of the Lie bracket of each fiber, i.e. if $\sigma, \sigma^{\prime} \in \Gamma(\operatorname{ad} P)$, then $\left[\sigma, \sigma^{\prime}\right](m)=\left[\sigma(m), \sigma^{\prime}(m)\right]$. It is possible, as discussed in [4, 14 and 15], to complete both $\mathscr{G}$ and $\mathbf{g}$ with respect to Sobolev norms so that $\mathscr{G}$ is a smooth infinite dimensional Lie group modeled on a Hilbert space and $\mathbf{g}$ is its Lie algebra. These issues will not be addressed in this paper.

The space of differential forms on $P$ with values in $\mathfrak{g}$ is denoted by $\Omega^{\bullet}(P, \mathfrak{g})$. An element $\varphi \in \Omega^{\bullet}(P, \mathfrak{g})$ is called horizontal if it is annihilated when contracted with a vertical vector field $X \in \mathscr{X}(P)_{V}$, while $\varphi$ is called $G$-equivariant if $R_{g}^{*}(\varphi)=\operatorname{Ad}\left(g^{-1}\right) \varphi$. The subspace of horizontal, $G$-equivariant forms is denoted by $\bar{\Omega}^{\bullet}(P, \mathfrak{g})^{\text {Ad }}$. It can be shown, as in [3 and 8], that $\bar{\Omega}^{\bullet}(p, \mathfrak{g})^{\text {Ad }}$ is isomorphic to $\Omega^{\bullet}(M$, ad $P)$, the differential forms on $M$ with values in $\Gamma($ ad $P)$.

Both $\Omega^{\bullet}(P, \mathfrak{g})$ and $\Omega^{\bullet}(M$, ad $P)$ are graded Lie algebras where the bracket in the first case is given by the Lie bracket of $\mathfrak{g}$ and the wedge of forms on $P$ and in the latter case by the Lie bracket of $\Gamma(\operatorname{ad} P)$ and the wedge of forms on $M$. The two brackets are equivalent when $\Omega^{\bullet}(M$, ad $P)$ is viewed as a Lie subalgebra of $\Omega^{\bullet}(P, \mathfrak{g})$. The bracket of $\Omega^{\bullet}(M$, ad $P)$ satisfies the graded identity $[\phi, \varphi]=(-1)^{p q+1}[\phi, \varphi]$, for $\phi \in \Omega^{p}(M$, ad $P), \varphi \in \Omega^{q}(M$, ad $P)$ and the graded Jacobi identity $(-1)^{r p}[\phi,[\varphi, \psi]]+(-1)^{p q}[\varphi,[\psi, \phi]]+(-1)^{q r}[\psi,[\phi, \varphi]]$ $=0$, for $\phi \in \Omega^{p}(M$, ad $P), \varphi \in \Omega^{q}(M$, ad $P)$ and $\psi \in \Omega^{r}(M$, ad $P)$. These graded relations also hold for $\Omega^{\bullet}(P, \mathfrak{g})$.

One of the many equivalent definitions of a connection $A$ on $P$ is that $A$ is a form in $\Omega^{1}(P, \mathfrak{g})$ such that $R_{g}^{*}(A)=\operatorname{Ad}\left(g^{-1}\right) A$ for all $g \in G$, and $A(s(x))=$ $x$, where $s(x)$ is the fundamental vector field on $P$ associated to $x \in \mathfrak{g}$. Recall that the vector field $s(x)$ is defined at $p \in P$ by $s(x)_{p}=\left.\frac{d}{d t} p \cdot \exp (t x)\right|_{t=0}$. Although a connection $A$ is a $G$-equivariant form, it is not horizontal, since it 
is not annihilated by fundamental vector fields, which are vertical. Note that the difference of two connections $A_{0}$ and $A_{1}$ is a horizontal, $G$-equivariant form, and thus $A_{1}-A_{0}$ can be regarded as an element of $\Omega^{1}(M$, ad $P$ ) for any two connections, $A_{0}, A_{1}$.

Given a connection $A$, the covariant derivative,

$$
d_{A}: \Omega^{p}(M, \text { ad } P) \rightarrow \Omega^{p+1}(M, \text { ad } P),
$$

induced by $A$ is defined by $d_{A}(\varphi)=d_{M} \varphi+[A, \varphi]$ for $\varphi \in \Omega^{p}(M$, ad $P)$, where $d_{M}$ is the exterior derivative of $M$.

1.2. Anomalies as cohomology classes. Let $\mathscr{A}$ be the space of all connections on a principal bundle $P$. A $C^{\infty}$ functional on $\mathscr{A}$ is a smooth map $\widetilde{\Psi}: \mathscr{A} \rightarrow$ $\mathbb{R}$, where the topology on $\mathscr{A}$, as for $\mathscr{G}$ and $\mathbf{g}$, is given by completing $\mathscr{A}$ relative to Sobolev norms. The space of all such functionals will be denoted by $C^{\infty}(\mathscr{A})$. In what follows, whenever the issue arises of what $C^{\infty}$ means, it is assumed that the appropriate iopologies have been defined via completion relative to Sobolev norms.

In [3], L. Bonora and P. Cotta-Ramusino describe the physical motivations for considering the graded algebra, $\Lambda^{\bullet}\left(\mathbf{g}, C^{\infty}(\mathscr{A})\right)$, of skew symmetric, multilinear maps of $\mathbf{g}$ into functionals of the space of connections $\mathscr{A}$ of $P$. In the terminology of physics, a connection $A$ is called a gauge vector potential while an element $\widetilde{\mathscr{L}}$ of $\Lambda^{p}\left(\mathbf{g}, C^{\infty}(\mathscr{A})\right)$ is said to be a functional of the gauge vector potentials with $p$ ghosts.

This graded algebra has a differential, $\delta: \Lambda^{p}\left(\mathbf{g}, C^{\infty}(\mathscr{A})\right) \rightarrow \Lambda^{p+1}\left(\mathbf{g}, C^{\infty}(\mathscr{A})\right)$, defined as follows. Since $\mathbf{g}=\Gamma(\operatorname{ad} P)$, it follows that $\mathbf{g}$ is isomorphic to $\Omega^{0}(M$, ad $P)$. But $\mathscr{A}$ is an affine space modeled on $\Omega^{1}(M$, ad $P)$. This implies, as for any affine space, that the tangent space of $\mathscr{A}$ at $A \in \mathscr{A}$, denoted by $T_{A}, \mathscr{A}$, is isomorphic to the model space $\Omega^{1}(M$, ad $P)$. Thus for each $A \in \mathscr{A}$, it follows that $d_{A}: \Omega^{0}(M$, ad $P) \rightarrow \Omega^{1}(M$, ad $P)$. This allows the definition of the differential $\delta: \Lambda^{p}\left(\mathbf{g}, C^{\infty}(\mathscr{A})\right) \rightarrow \Lambda^{p+1}\left(\mathbf{g}, C^{\infty}(\mathscr{A})\right)$. If the evaluation of $\widetilde{\mathscr{L}} \in \Lambda^{p}\left(\mathbf{g}, C^{\infty}(\mathscr{A})\right)$ at $f_{1}, \ldots, f_{p} \in \mathbf{g}$ and $A \in \mathscr{A}$ is denoted by $\widetilde{\mathscr{L}}\left(f_{1}, \ldots, f_{p}, A\right)$, then the differential $\delta$ is defined by

$$
\begin{aligned}
(\delta \widetilde{\mathscr{L}}) & \left(f_{1}, \ldots, f_{p+1}, A\right) \\
= & \sum_{i=1}^{p+1}(-1)^{i+1} \theta\left(f_{i}\right)\left(\widetilde{\mathscr{L}}\left(f_{1}, \ldots, \hat{f}_{i}, \ldots, f_{p+1}, A\right)\right) \\
& +\sum_{i<j}(-1)^{i+j} \widetilde{\mathscr{L}}\left(\left[f_{i}, f_{j}\right], f_{1}, \ldots, \hat{f}_{i}, \ldots, \hat{f}_{j}, \ldots, f_{p+1}, A\right),
\end{aligned}
$$

where $f_{i} \in \mathbf{g}, \hat{f}_{i}$ means that $f_{i}$ has been omitted, and

$$
\begin{aligned}
\theta\left(f_{i}\right) & \left(\widetilde{\mathscr{L}}\left(f_{1}, \ldots, \hat{f}_{i}, \ldots, f_{p+1}, A\right)\right) \\
& =-\left.\frac{d}{d t} \widetilde{\mathscr{L}}\left(f_{1}, \ldots, \hat{f}_{i}, \ldots, f_{p+1}, A+t d_{A} f_{i}\right)\right|_{t=0}
\end{aligned}
$$

is the negative of the derivative of $\widetilde{\mathscr{L}}$ along the line $A+t d_{A} f_{i}$ in $\mathscr{A}$ defined by $d_{A} f_{i} \in T_{A} \mathscr{A}$. Note that for all $t$ it follows that $A+t d_{A} f_{i} \in \mathscr{A}$ and thus the evaluation of $\widetilde{\mathscr{L}}$ at $A+t d_{A} f_{i}$ makes sense. This generalizes, on the one hand, 
the exterior derivative $d$ of differential forms on a manifold $M$, where $\theta(f)$ takes the place of the Lie derivative of a form along a vector field of $M$ and, on the other hand, the differential of the Chevalley-Eilenberg complex of $\mathfrak{g}$ where $\theta(f)$ takes the place of the derivation $\theta(x)$. To show that $\delta$ is a differential, i.e. that $\delta^{2}=0$, is analogous to showing that $d^{2}=0$ for the exterior derivative or $\delta^{2}=0$ for the Chevalley-Eilenberg differential. Of course the cohomology of this complex is defined by, $H_{\delta}^{n}\left(\mathbf{g}, C^{\infty}(\mathscr{A})\right)=\operatorname{ker} \delta / \operatorname{im} \delta$. The anomalies of physics lie in $H^{1}\left(\mathbf{g}, C^{\infty}(\mathscr{A})\right)$.

1.3. Examples. The following are four examples from the literature of anomalies described as cohomology classes. The importance of these examples is neither their technical details nor their differences but their similarity. Although the specific examples depend upon the dimension of the base space $M$, the property which they all share is that the anomalies are represented by integrals over $M$ of invariantly defined forms, i.e. integrands which are globally defined without reference to partitions of unity nor coordinate charts. The integrand in all the examples is an $n$-form defined by a symmetric ad-invariant polynomial, $P_{k} \in S^{k}(\mathfrak{g})^{\text {ad }}$, evaluated at $f \in \mathbf{g}$, and at terms involving $A$ and its curvature $F$. In the rest of this paper, it is the invariance of these integrands which will be explored in the setting which gives rise to the first example.

Example 1. In [3], L. Bonora and P. Cotta-Ramusino describe the representatives of the anomalies by integrating over $M$ representatives of the total cohomology classes of the local bicomplex. The local bicomplex, which will be described in detail at the end of this chapter, is constructed through the use of a fixed connection $A_{0} \in \mathscr{A}$. In particular, note that $A-A_{0} \in \Omega^{1}(M$, ad $P)$ for all $A \in \mathscr{A}$. Given a symmetric ad-invariant multilinear map, then there is a naturally defined symmetric $k$-multilinear map, also denoted $P_{k}$,

$$
P_{k}: \Omega^{i_{1}}(M, \text { ad } P) \times \cdots \times \Omega^{i_{k}}(M, \text { ad } P) \rightarrow \Omega^{i_{1}+\cdots+i_{k}}(M),
$$

namely if $\omega_{i_{n}} \in \Omega^{i_{n}}(M$, ad $P)$ then $P_{k}\left(\omega_{i_{1}}, \ldots, \omega_{i_{k}}\right) \in \Omega^{i_{1}+\cdots+i_{k}}(M)$. Note that if $i_{1}+\cdots+i_{k}=n$, then the resulting $n$-form is integrable over $M$. It is from these $P_{k}$, evaluated at $f \in \Omega^{0}(M$, ad $P), A-A_{0} \in \Omega^{1}(M$, ad $P)$, and $d_{M}\left(A-A_{0}\right) \in \Omega^{2}(M$, ad $P) \quad\left(d_{M}\right.$ is the exterior derivative of $\left.M\right)$, that the representatives of the total cohomology classes are constructed. Two specific examples involving $P_{2}$ and $P_{3}$ are the following: (1) when $M$ is two dimensional, then the representative is given by

$$
\int_{M} P_{2}\left(f, d_{M}\left(A-A_{0}\right)\right)
$$

and (2) when $M$ is four dimensional, then the representative is given by

$$
\begin{aligned}
\int_{M} P_{3}\left(f, d_{M}\left(A-A_{0}\right), d_{M}\left(A-A_{0}\right)\right) \\
\quad+\frac{1}{4} \int_{M} P_{3}\left(f, d_{M}\left(A-A_{0}\right),\left[A-A_{0}, A-A_{0}\right]\right) \\
\quad-\frac{1}{2} \int_{M} P_{3}\left(f, A-A_{0},\left[d_{M}\left(A-A_{0}\right), A-A_{0}\right]\right) .
\end{aligned}
$$

Note that in each case the integrand is of the appropriate dimension and that the dimension $n$ of $M$ is equal to $2 k-2$. The integrands, in both cases, are 
$n$-forms on $M$ which depend on $A \in \mathscr{A}$ and $f \in \mathbf{g}$. These types of $n$-forms will be denoted by $\Lambda^{1}\left(\mathbf{g}, C^{\infty}\left(\mathscr{A}, \Omega^{n}(M)\right)\right)$ to foreshadow the local bicomplex.

Example 2. In [5], P. Cotta-Ramusino describes the following two examples: (1) the Adler-Bell-Bardeen-Jackiw anomaly of $2 k-2$ dimensional manifolds $M$ is represented by

$$
\int_{M}\left(k(k-1) \int_{0}^{1}(1-t) P_{k}\left(d f, A, F_{t}, \ldots, F_{t}\right) d t\right),
$$

where $F_{t}=t F+\frac{1}{2}\left(t^{2}-t\right)[A, A], F$ being the curvature of $A$ and (2) the covariant anomaly for $2 k-2$ dimensional manifolds $M$ is represented by $\int_{M} k P_{k}(f, F, \ldots, F)$. Note that in each example the integrand is in $\Lambda^{1}\left(\mathbf{g}, C^{\infty}\left(\mathscr{A}, \Omega^{n}(M)\right)\right)$.

Example 3. In [17], R. Schmidt describes the representatives of anomalies for manifolds of dimensions $n=2 k-2$ by the following formula:

$$
\int_{M}\left(k(k-1) \int_{0}^{1}(1-t) \operatorname{Tr}\left(f \wedge d\left((A+f) \wedge\left(F_{t}\right)^{k-2}\right)\right) d t\right),
$$

where $F_{t}=t F+\left(t^{2}-t\right)((A+f) \wedge(A+f)), F$, again, being the curvature of $A, d$ in this setting is the total derivative of $\Lambda^{\bullet}\left(\mathbf{g}, C^{\infty}(\mathscr{A})\right)$, and the wedge product is the wedge of forms on $P$ with values in $\mathfrak{g}$. To show that the integrand is in $\Lambda^{1}\left(\mathbf{g}, C^{\infty}\left(\mathscr{A}, \Omega^{n}(M)\right)\right)$ requires some hard analysis of the infinite jet space of sections of the bundles in question.

Example 4. In [2], M. F. Atiyah and I. M. Singer give a formula for the representative of a primitive element $t_{2 j-1} \in H^{2 j-1}(\mathscr{G}, \mathbb{R})$, where $\mathscr{G}$ has been restricted to the gauge transformations which leave a point of $P$ fixed (the projection of this point in $M$ is the point at "infinity") and $H^{\bullet}(\mathscr{G}, \mathbb{R})$ is the cohomology of this restricted gauge group with coefficients in $\mathbb{R}$. This cohomology can be derived from $H^{\bullet}\left(\mathbf{g}, C^{\infty}(\mathscr{A})\right)$ by restricting the functionals on $\mathscr{A}$ to orbits of $\mathscr{G}$ through a fixed connection $A \in \mathscr{A}$ and viewing $\mathbf{g}$ as leftinvariant vector fields on $\mathscr{G}$. The action of $\mathscr{G}$ on $\mathscr{A}$ is just pulling back the connection $A \in \mathscr{A}$ by the diffeomorphisms $\boldsymbol{\theta} \in \mathscr{G}$, i.e. $\boldsymbol{\Theta} \cdot A=\Theta^{*}(A)$ and because of the above mentioned restriction this action is free and the orbits are isomorphic to $\mathscr{G}$.

Let $M$ have dimension $2 n$ and let $\alpha_{2 j+2 n-1}$ be defined as

$$
\alpha_{2 j+2 n-1}(A)=(j+n) \int_{0}^{1} P_{j+n}\left(A, F_{t}, \ldots, F_{t}\right) d t,
$$

where $F_{t}$ is defined as in Example 2. The formula for $t_{2 j-1}$ at $\Theta \in \mathscr{G}$ is given by

$$
t_{2 j-1}\left(f_{1}, \ldots, f_{2 j-1}\right)=\int_{M} i\left(f_{1}, \ldots, f_{2 j-1}\right) \alpha_{2 j+2 n-1}(\boldsymbol{\Theta} \cdot A),
$$

where $\alpha_{2 j+2 n-1}$ is interpreted as a $2 j+2 n-1$-form on $M \times \mathscr{G}$ and the interior product of $f_{1}, \ldots, f_{2 j-1}$ with $\alpha_{2 j+2 n-1}$ is $i\left(f_{1}, \ldots, f_{2 j-1}\right)$. Note that when $j=1$, i.e. when $t_{1}$ is in $H^{1}(\mathscr{G}, \mathbb{R})$, then the polynomial $P_{1+n}$ satisfies the 
relation $2(1+n)-2=2 n$ and the formula becomes

$$
t_{1}(f)=\int_{M}\left(i(f) \int_{0}^{1} P_{1+n}\left(\Theta \cdot A, \Theta \cdot F_{t}, \ldots, \Theta \cdot F_{t}\right) d t\right),
$$

which strongly resembles the formulas of the preceding examples.

This is not an exhaustive list of all the formulas and examples which appear in the literature, but it does represent several of the main perspectives taken in the description of anomalies as cohomology classes. The rest of this paper will explore the setting of the first example.

1.4. The local bicomplex. In [3], the authors, motivated by physical considerations, restrict the type of functionals in $C^{\infty}(\mathscr{A})$. The only functionals which will be considered are those which depend on $A \in \mathscr{A}$ in a "local" manner. Before describing precisely what type of functionals these are, local must be defined.

A multilinear operator $\widetilde{\mathscr{L}}$ mapping the sections of one vector bundle to the sections of another is called local if it decreases support. Thus if $V$ and $W$ are vector bundles over a manifold $M$, a multilinear operator, $\widetilde{\mathscr{L}}: \Gamma(V)^{p} \rightarrow \Gamma(W)$, is local if

$$
\operatorname{Supp} \widetilde{\mathscr{L}}\left(\xi_{1}, \ldots, \xi_{p}\right) \subset \bigcap_{i=1}^{p} \operatorname{Supp}\left(\xi_{i}\right),
$$

where $\Gamma(V)^{p}=\Gamma(V) \times \cdots \times(V) p$ times, $\xi_{i} \in \Gamma(V), \operatorname{Supp}\left(\xi_{i}\right)=\{m \in M \mid$ $\left.\xi_{i}(m) \neq 0\right\}$, and $\operatorname{Supp} \widetilde{\mathscr{L}}\left(\xi_{1}, \ldots, \xi_{p}\right)=\left\{m \in M \mid \widetilde{\mathscr{L}}\left(\xi_{1}(m), \ldots, \xi_{p}(m)\right) \neq\right.$ $0\}$. The crucial theorem due to J. Peetre [13] states that a local operator $\widetilde{\mathscr{L}}$ is a differential operator in local coordinates.

The restricted functionals in $C^{\infty}(\mathscr{A})$ which will be considered are as follows. Let $\Psi_{m}$ be a local symmetric $m$-multilinear operator from $\Omega^{1}(M \text {, ad } P)^{m}$ to $\Omega^{n}(M)$. The set of these operators will be denoted by $S^{m}\left(\Omega^{1}(M\right.$, ad $\left.P), \Omega^{n}(M)\right)$. Since, by Peetre's theorem, $\Psi_{m}$ is a differential operator in local coordinates, it follows that in local coordinates $\Psi_{m}$ is a homogeneous polynomial of degree $m$ in the derivatives of elements of $\Omega^{1}\left(M\right.$, ad $P$ ) with values in $\Omega^{n}(M)$. The space of all such polynomials will be denoted by $S^{\bullet}\left(\Omega^{1}(M\right.$, ad $\left.P), \Omega^{n}(M)\right)$. Thus a typical element $\Psi \in S^{\bullet}\left(\Omega^{1}(M\right.$, ad $\left.P), \Omega^{n}(M)\right)$ is of the form $\Psi=$ $\sum_{m}^{\text {finite }} \Psi_{m}$, where $\Psi_{m}$ is in $S^{m}\left(\Omega^{1}(M\right.$, ad $\left.P), \Omega^{n}(M)\right)$.

Given a fixed connection $A_{0} \in \mathscr{A}$, called a background connection in the physics literature, an element $\tilde{\Psi}$ of $C^{\infty}(\mathscr{A})$ can be defined by

$$
\tilde{\Psi}(A)=\int_{M}\left(\sum_{m}^{\text {finite }} \Psi_{m}(\underbrace{A-A_{0}, \ldots, A-A_{0}}_{m})\right),
$$

since $A-A_{0} \in \Omega^{1}(M$, ad $P)$ for all connections $A$. In what follows Chern's convention for evaluation will be used, i.e. if there are not enough arguments to fill a multilinear operator then the last argument is repeated the required numbers of times. Thus, by this convention, it follows that $\Psi_{m}\left(A-A_{0}\right)=$ $\Psi_{m}\left(A-A_{0}, \ldots, A-A_{0}\right)$, where $A-A_{0}$ occurs $m$ times, and hence the formula $\widetilde{\Psi}(A)=\int_{M} \Psi\left(A-A_{0}\right)=\int_{M} \sum_{m}^{\text {finite }} \Psi_{m}\left(A-A_{0}\right)$ is well defined. These are the functionals in $C^{\infty}(\mathscr{A})$ which are said to depend on $A$ in a local manner. They 
will be denoted by $\operatorname{Pol}_{A_{0}}(\mathscr{A})$ to reflect that they are defined via the background connection $A_{0}$ by integrating elements of $S^{\bullet}\left(\Omega^{1}(M\right.$, ad $\left.P), \Omega^{n}(M)\right)$ over $M$.

The complex $\Lambda^{\bullet}\left(\mathbf{g}, C^{\infty}(\mathscr{A})\right)$ contains the subcomplex $\Lambda^{\bullet}\left(\mathbf{g}, \operatorname{Pol}_{A_{0}}(\mathscr{A})\right)$, where all operators are restricted to be local. Thus, if $\widetilde{\mathscr{L}}$ is in $\Lambda^{\bullet}\left(\mathbf{g}, \operatorname{Pol}_{A_{0}}(\mathscr{A})\right)$, then $\widetilde{\mathscr{L}}$ is a local skew-symmetric $p$-multilinear operator from $\mathbf{g}$ to $S^{\bullet}\left(\Omega^{1}(M\right.$, ad $\left.P), \Omega^{n}(M)\right)$ and its evaluation at $A \in \mathscr{A}$ is defined via the background connection. To emphasize this dependency on the background connection, $\widetilde{\mathscr{L}}$ evaluated at $f_{1}, \ldots, f_{p} \in \mathbf{g}$ and $A \in \mathscr{A}$ will be written as $\widetilde{\mathscr{L}}\left(f_{1}, \ldots, f_{p+1}, A-A_{0}\right)$.

Since the covariant derivative determined by a connection $A$ is given by $d_{A} f=d f+[A, f]$ for $f \in \mathbf{g}$, it follows that $d_{A} f=d_{A_{0}} f+\left[A-A_{0}, f\right]$. Thus the action of $\theta$, used to define the differential $\delta$ of the larger complex $\Lambda\left(\mathbf{g}, C^{\infty}(\mathscr{A})\right)$, preserves this subcomplex, since

$$
\begin{aligned}
\theta\left(f_{i}\right) & \widetilde{\mathscr{L}}\left(f_{1}, \ldots, \hat{f}_{i}, \ldots, f_{p+1}, A-A_{0}\right) \\
& =-\left.\frac{d}{d t} \widetilde{\mathscr{L}}\left(f_{1}, \ldots, \hat{f}_{i}, \ldots, f_{p+1},\left(A-A_{0}\right)+t d_{A} f_{i}\right)\right|_{t=0} \\
& =-\frac{d}{d t} \widetilde{\mathscr{L}}\left(f_{1}, \ldots, \hat{f}_{i}, \ldots, f_{p+1},\left(A-A_{0}\right)+\left.t\left(d_{A_{0}} f_{i}+\left[A-A_{0}, f_{i}\right]\right)\right|_{t=0}\right.
\end{aligned}
$$

and hence the dependency on $A$ is still by the difference of $A$ and $A_{0}$. Consequently the differential $\delta$ of the original complex restricts to the subcomplex and the cohomology of the subcomplex is well defined.

The local bicomplex results from relaxing the condition that the local operators $\Psi$ have values in $\Omega^{n}(M)$. Thus, in general, an operator $\Psi^{q}$ has values in $\Omega^{q}(M)$ for $0 \leq q \leq n$ and as before $\Psi^{q}=\sum_{m}^{\text {finite }} \Psi_{m}^{q}$, where $\Psi_{m}^{q} \in S^{m}\left(\Omega^{1}(M\right.$, ad $\left.P), \Omega^{q}(M)\right)$. Through the use of the background connection, these are $q$-forms on $M$ which depend on $A \in \mathscr{A}$ in a local manner. The bicomplex itself is $\Lambda^{p}\left(\mathbf{g}, S\left(\Omega^{1}(M\right.\right.$, ad $\left.P), \Omega^{q}(M)\right)$, which will be denoted by $S_{M}^{p, q}$. An element of $S_{M}^{p, q}$ is said to have vertical degree $p$ and form degree $q$ (in the physics literature the vertical degree is the ghost degree). Note that if $\mathscr{L}$ is in $S_{M}^{p, q}$, then $\mathscr{L}=\sum_{m}^{\text {finite }} L_{m}$ where $L_{m} \in \Lambda^{q}\left(\mathbf{g}, S^{m}\left(\Omega^{1}(M\right.\right.$, ad $\left.P), \Omega^{q}(M)\right)$. Again using Chern's convention, if $\mathscr{L}$ is in $S_{M}^{p, q}$, then for $f_{1}, \ldots, f_{p} \in \mathbf{g}$ and $A \in \mathscr{A}$, the formula,

$$
\mathscr{L}\left(f_{1}, \ldots, f_{p}, A-A_{0}\right)=\sum_{m}^{\text {finite }} L_{m}\left(f_{1}, \ldots, f_{p}, A-A_{0}\right),
$$

is well defined. In particular, if $\mathscr{L}$ is in $S_{M}^{p, n}$, then $\int_{M} \mathscr{L} \in \Lambda^{p}\left(\mathbf{g}, \operatorname{Pol}_{A_{0}}(\mathscr{A})\right)$. The differential $\delta$ of $\Lambda^{\bullet}\left(\mathrm{g}, \mathrm{Pol}_{A_{0}}(\mathscr{A})\right)$ induces a differential, also denoted $\delta$, on the bicomplex $S_{M}^{\bullet}, \bullet$. It is defined exactly as for $\Lambda^{\bullet}\left(\mathbf{g}, \operatorname{Pol}_{A_{0}}(\mathscr{A})\right)$ and thus $\delta: S_{M}^{p, q} \rightarrow S_{M}^{p+1, q}$. If $M$ is compact and oriented, then the differential $\delta$ commutes with integration, i.e. $\delta\left(\int_{M} \mathscr{L}\right)=\int_{M}(\delta \mathscr{L})$, for $\mathscr{L}$ in $S_{M}^{p, n}$. by

The bicomplex $S_{M}^{\bullet}{ }^{\bullet}$ has a second differential, denoted by $\partial$. It is defined

$$
(\partial \mathscr{L})\left(f_{1}, \ldots, f_{p}, A-A_{0}\right)=d_{M}\left(\mathscr{L}\left(f_{1}, \ldots, f_{p}, A-A_{0}\right)\right),
$$

where $d_{M}$ is the exterior derivative of $M$, and thus $\partial: S_{M}^{p, q} \rightarrow S_{M}^{p, q+1}$. 
In what follows the variable $\omega$ will be used for $A-A_{0}$. Thus $\omega$ is the element of $\Omega^{1}(M$, ad $P)$ determined by $A-A_{0}$ and as $A$ varies through $\mathscr{A}, \omega$ varies through $\Omega^{1}(M$, ad $P)$.

It is noted in [3] that $\delta \partial=\partial \delta$ and hence there is the following commutative diagram of complexes:

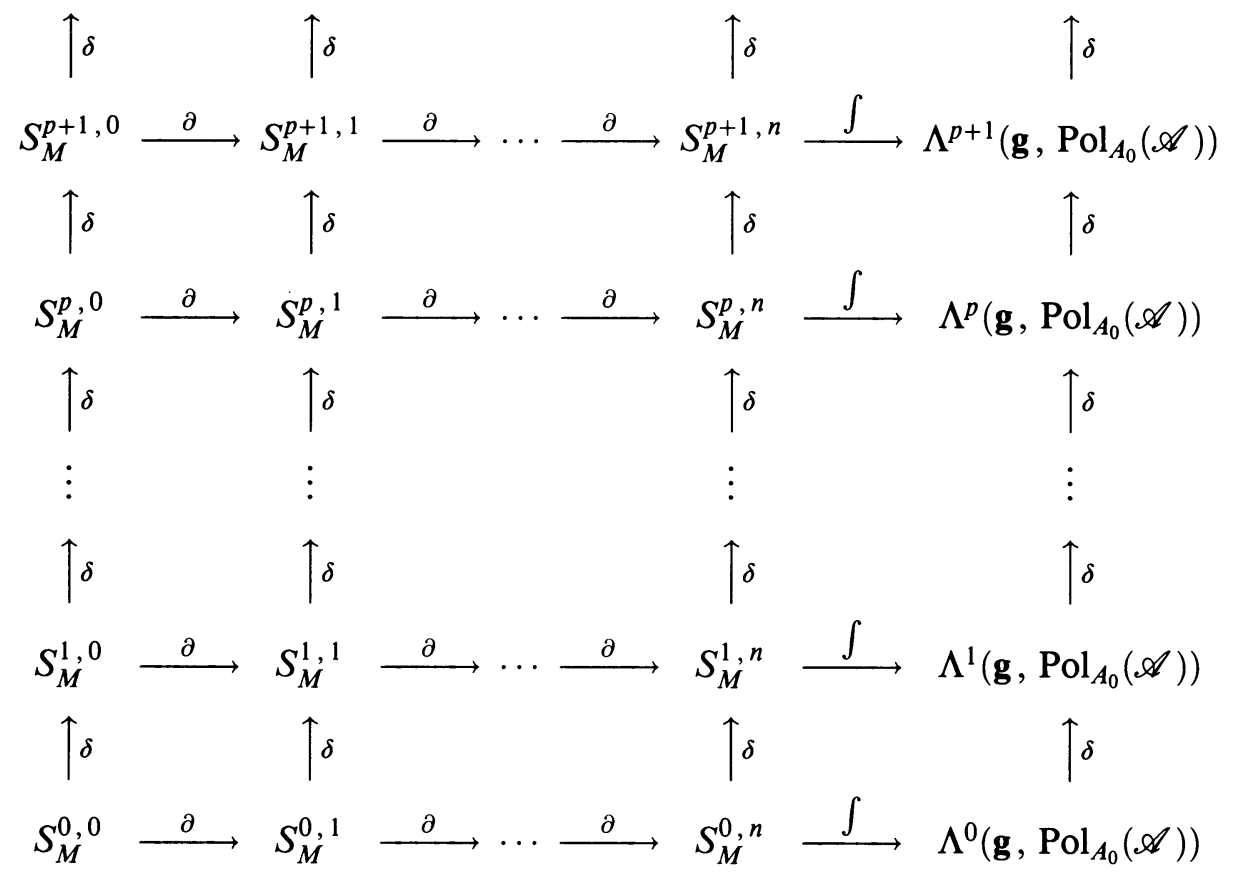

Since $\delta \partial=\partial \delta$, the bicomplex $S_{M}^{\bullet, \bullet}$ has a total differential, $d$, defined by $d=\partial+(-1)^{q} \delta$. The representatives of the anomalies in [3] are integrals over $M$ of representatives of total cohomology classes in $H_{d}^{n+1}\left(S_{M}^{\bullet} \cdot \bullet\right)$.

\section{OVERVIEW}

This paper starts with the above operators expressed as differential operators in local coordinates, determines what conditions the operators must satisfy to be independent of this expression, and then computes the cohomology of the resulting invariantly defined local differential operators.

The first step will be to examine the operators' descriptions over local trivializations of the principal fiber bundle $P$. Thus, if $U$ is an open set in the base manifold $M$, the objective is to describe $\left.S_{M}^{\bullet, \bullet}\right|_{U}$ in the setting of the trivial bundle $U \times G$. In other words, the operators will be written explicitly as differential operators of the sections of the locally trivialized bundles. This is possible because of the assumption of locality and Peetre's theorem. The operators thus expressed will be denoted by $S_{U}^{\bullet} \cdot \bullet$.

Once this description is accomplished, those operators which are independent of their coordinate expression will be found by determining which are invariant under the action of the pseudo-group of local diffeomorphisms of $\mathbb{R}^{n}$, denoted $\operatorname{Diff}_{\text {loc }}\left(\mathbb{R}^{n}\right)$. These operators will be denoted by $\left(S_{U}^{\bullet} \bullet\right)^{\text {Diff }}$ oc $\left(\mathbb{R}^{n}\right)$.

When the invariant operators are found, the differentials of $S_{M}^{\bullet, \bullet}$ will be translated to $\left(S_{U}^{\bullet, \bullet}\right)^{\text {Diff }}$ loc $\left(\mathbb{R}^{n}\right)$. The cohomology of the invariants will then be 
computed. The main theorem in $\S 9$ states that

$$
H_{d}^{\bullet}\left(\left(S_{U}^{\bullet}, \bullet\right)^{\operatorname{Diff}_{\text {loc }}\left(\mathbb{R}^{n}\right)}\right) \simeq H_{d}^{\bullet}\left(W(\mathfrak{g})_{\left[\frac{n}{2}\right]}\right),
$$

where $W(\mathfrak{g})_{\left[\frac{n}{2}\right]}$ is the Weil algebra of $\mathfrak{g}$, the Lie algebra of $G$, truncated at $\left[\frac{n}{2}\right]$ the greatest integer less than or equal to half the dimension of the base space $M$. This implies that $H_{d}^{q}$ is 0 if $q<n$ or $n+r<q$ and that $\operatorname{dim}\left(H_{d}^{q}\right)<\infty$ when $n \leq q \leq n+r$, since this is true of the cohomology of the truncated Weil algebra.

Finally, this result will be globalized in the case that $\operatorname{ad}^{*} P$ is a trivializable bundle.

\section{Algebraic PRELIMinaries}

The purpose of this section is to recall the relevant algebraic structures of differential graded vector spaces, differential graded algebras, and $\mathfrak{g}$-differential graded algebras, where $\mathfrak{g}$ is the Lie algebra of a compact, connected Lie group $G$. This will, among other things, establish the sign conventions which will be used through the rest of the paper. Two examples which will play important roles in what follows will be described, namely, the Koszul algebra, $K(V)$, of a graded vector space $V$ and the Weil algebra, $W(\mathfrak{g})$, of a Lie algebra $\mathfrak{g}$. The former is an example of a universal differential graded algebra, while the latter is an example of a universal $\mathfrak{g}$-differential graded algebra with a connection. In all that follows $k$ denotes a field of characteristic 0 .

3.1. Graded vector spaces. A graded vector space $V$ is the direct sum of a collection of vector spaces $\left\{V^{n}\right\}$, indexed by the integers $n \in \mathbb{Z}$; it is denoted by $\bigoplus_{n \in \mathbb{Z}} V^{n}=V$. An element $v \in V^{n}$ is called homogeneous of degree $n$. In general, the degree of a homogeneous element will be denoted by $\operatorname{deg}(v)$. It is useful to consider the following decomposition of a graded vector space. By definition, the even subspace of a graded vector space is $V^{\text {even }}=\bigoplus_{n \equiv 0 \bmod 2} V^{n}$ and the odd subspace is $V^{\text {odd }}=\bigoplus_{n \equiv 1 \bmod 2} V^{n}$. Thus the graded vector space $V$ is also the direct sum $V=V^{\text {odd }} \oplus V^{\text {even }}$.

Given two graded vector spaces $V$ and $W$, then their direct sum and their tensor product are graded vector spaces as well, where $(V \oplus W)^{n}=V^{n} \oplus W^{n}$ and $(V \otimes W)^{n}=\bigoplus_{p+q=n}\left(V^{p} \otimes W^{q}\right)$. Suppose that $V$ and $W$ are two graded vector spaces. A linear map $\phi: V \rightarrow W$ is called graded, if there exists an integer $n$, called the degree of $\phi$, such that $\phi: V^{m} \rightarrow W^{m+n}$. The space of all graded linear maps is denoted by $\operatorname{Hom}(V, W)$. The vector space $\operatorname{Hom}(V, W)$ is itself a graded vector space, where $\operatorname{Hom}(V, W)^{n}$ consists of the graded linear maps of degree $n$. If $V, V^{\prime}, W$, and $W^{\prime}$ and four graded vector spaces and $\phi \in \operatorname{Hom}(V, W), \psi \in \operatorname{Hom}\left(V^{\prime}, W^{\prime}\right)$, then $\phi \otimes \psi$ in $\operatorname{Hom}\left(V \otimes V^{\prime}, W \otimes W^{\prime}\right)$ is defined by $(\phi \otimes \psi)\left(v \otimes v^{\prime}\right)=(-1)^{\operatorname{deg}(\psi) \cdot \operatorname{deg}(v)} \phi(v) \otimes \psi\left(v^{\prime}\right)$. This generalizes the evaluation of tensor products to the graded setting.

A differential graded vector space is a graded vector space $V$ with a linear map $\delta$, called a differential, such that $\delta: V^{n} \rightarrow V^{n+1}$, for all $n \in \mathbb{Z}$, and $\delta^{2}=0$. The cohomology of a differential graded vector space is defined by $H_{\delta}^{\bullet}(Y)=\operatorname{ker} \delta / \operatorname{im} \delta$, where $\delta$ has been included as a subscript to indicate that the cohomology has been computed respect to $\delta$.

3.2. Graded algebras. A graded algebra $A$ is a graded vector space with an associative multiplication, a bilinear map $A \times A \rightarrow A$, such that, if $a \in A^{p}$ 
and $a^{\prime} \in A^{q}$ then $a \cdot a^{\prime} \in A^{p+q}$ and $\left(a \cdot a^{\prime}\right) \cdot a^{\prime \prime}=a \cdot\left(a^{\prime} \cdot a^{\prime \prime}\right)$. All algebras are assumed to be unitary and thus $k \subset A^{0}$, where $k$ is the ground field of $A$. An element $a \in A^{n}$ is said to be homogeneous of degree $n$. If, for all $a \in A^{p}$ and all $a^{\prime} \in A^{q}, a \cdot a^{\prime}=(-1)^{p \cdot q} a^{\prime} \cdot a$, then the graded algebra is called graded commutative.

Given a graded vector space $\bigoplus_{n \in \mathbb{Z}} V^{n}=V$, there exists a free graded commutative algebra, denoted by $\Lambda\left(\bigoplus_{n \in \mathbb{Z}} V^{n}\right)=\Lambda(V)$, and an inclusion, a degree zero graded vector space homomorphism, $l: \bigoplus_{n \in \mathbb{Z}} V^{n} \rightarrow \Lambda(V)$, which satisfies the following universal property. Given any graded commutative algebra $A$ and a degree zero graded vector space homomorphism, $\psi: V \rightarrow A$, then there exists a unique graded algebra homomorphism, $\tilde{\psi}: \Lambda(V) \rightarrow A$, of degree zero, such that $\psi=\tilde{\psi} \circ l$. The algebra $\Lambda(V)$ is a free graded commutative algebra, with the elements of $V$ as generators. By an abuse of notation, the product of $\Lambda(V)$ will be denoted by $\wedge$.

In general there is an isomorphism $\Lambda(V) \simeq E\left(V^{\text {odd }}\right) \otimes S\left(V^{\text {even }}\right)$, where $E$ is used to reflect the anticommuting or exterior multiplication and $S$ is used to reflect the commuting or symmetric multiplication of their respective elements. Both $E(V)$ and $S(V)$ are graded algebras. Finally, there is a canonical isomorphism of graded commutative algebras $\Lambda(V \oplus W) \simeq \Lambda(V) \otimes \Lambda(W)$.

As an example, one which will be used frequently in what follows, consider the suspension of $\sigma V$ of a vector space $V$, where $V$ is defined to be of degree one. As a vector space $\sigma V$ is isomorphic to $V$ but $\operatorname{deg}(\sigma v)=2$. Consider the graded vector space $V \oplus \sigma V$, so that $(V \oplus \sigma V)^{1}=V,(V \oplus \sigma V)^{2}=\sigma V$, and $(V \oplus \sigma V)^{i}=0$ for all $i \neq 1,2$. In this case, $\Lambda(V \oplus \sigma V)$ is easy to describe. Clearly $\Lambda(V \oplus \sigma V)$ is just $E(V) \otimes S(\sigma V)$ and it is easily shown that $\Lambda^{n}(V \oplus \sigma V)=\bigoplus_{p+2 q=n} E^{p}(V) \otimes S^{q}(\sigma V)$.

3.3. Graded Lie algebras and differential graded algebras. A graded Lie algebra is a graded vector space $L$ with a bracket [, ], a bilinear map such that: $\left[L^{p}, L^{q}\right] \subset L^{p+q},[x, y]=(-1)^{\operatorname{deg}(x) \cdot \operatorname{deg}(y)+1}[y, x]$, and

$$
[x,[y, z]]=[[x, y], z]+(-1)^{\operatorname{deg}(x) \cdot \operatorname{deg}(y)}[y,[x, z]] .
$$

The latter is the graded Jacobi identity. An example of a graded Lie algebra is the space of graded derivations of a graded algebra $A$, denoted by $\operatorname{Der}(A)$. It consists of graded linear maps $D$ of the underlying graded vector space, such that

$$
D\left(a \cdot a^{\prime}\right)=D(a) \cdot a^{\prime}+(-1)^{\operatorname{deg}(D) \cdot \operatorname{deg}(a)} a \cdot D\left(a^{\prime}\right) .
$$

Note that the derivation property implies that $D(k)=0 . \operatorname{Der}(A)$ inherits the grading of the graded linear maps and the bracket of $\operatorname{Der}(A)$ is defined by

$$
\left[D, D^{\prime}\right]=D \circ D^{\prime}+(-1)^{\operatorname{deg}(D) \cdot \operatorname{deg}\left(D^{\prime}\right)+1} D^{\prime} \circ D \text {. }
$$

A differential graded algebra is a graded algebra $A$, with a graded derivation $\delta$ of degree +1 , such that $\delta^{2}=0$. Thus, $\delta$ is a differential for the underlying graded vector space. Again, the cohomology of a differential graded algebra $A$ is defined as usual; but in the setting of a graded algebra, $H_{\delta}^{\bullet}(A)$ inherits an algebra structure. The following is an important example of a differential graded algebra.

3.4. The Koszul algebra $K(V)$ of a graded vector space $V$. The Koszul algebra is the universal differential graded algebra, associated to a graded vector space 
$V$. Thus, $K(V)$ is the unique differential graded algebra with a degree zero homomorphism $l$ of graded vector spaces $l: V \rightarrow K(V)$, such that, given a degree zero graded vector space homomorphism $\Psi$ of $V$ to a differential graded algebra $A, \Psi: V \rightarrow A$, then there is a degree zero differential graded algebra homomorphism, $\widetilde{\Psi}: K(V) \rightarrow A$, with $\widetilde{\Psi} \circ l=\Psi$.

As a graded algebra, $K(V)$ is just $\Lambda(V \oplus \sigma V)$. In the setting of graded vector spaces, $\sigma V$, the suspension of $V$, is defined as the graded vector space such that $(\sigma V)^{n}$ is isomorphic to $V^{n-1}$. The differential $\partial$ of $K(V)$ is defined as follows. On $k \subset K(V)^{0}$ and for $\sigma v \in \sigma V, \partial$ is defined to be identically zero. For $v \in V, \partial$ is defined as $\partial(v)=\sigma v$. This is sufficient for a canonical extension of $\partial$ to a derivation of degree one on all of $\Lambda(V \oplus \sigma V)$. Thus $K(V)$ is a free differential graded commutative algebra on the generators $v$ and their differentials $\partial v=\sigma v$. Furthermore, as was shown by Koszul in [12], $K(V)$ is acyclic, i.e. $H_{\partial}^{n}(K(V))=0$, if $i>0$, and $H_{\partial}^{0}(K(V))=k$. The Koszul algebra which will be used in the rest of this paper is the Koszul algebra of the vector space $\mathfrak{g}^{*}$, where $\mathfrak{g}^{*}$ is the dual of a Lie algebra $\mathfrak{g}$ and $\mathfrak{g}^{*}$ is viewed as a graded vector space by setting $\left(\mathfrak{g}^{*}\right)^{1}=\mathfrak{g}^{*}$ and $\left(\mathfrak{g}^{*}\right)^{i}=0$ for $i \neq 1$. By an abuse of notation, to be consistent with the notation in the literature for the ChevalleyEilenberg complex and the Weil algebra of $\mathfrak{g}$, this will be denoted by $K(\mathfrak{g})$. Thus, $K(\mathfrak{g})$ will always mean the differential graded algebra $\Lambda\left(\mathfrak{g}^{*} \oplus \sigma \mathfrak{g}^{*}\right)$, with the differential $\partial$, such that $\partial(\eta)=\sigma \eta$ and $\partial(\sigma \eta)=0$.

3.5. $\mathfrak{g}$-differential graded algebras. The following definition is given by Cartan in [4]. A $\mathfrak{g}$-differential graded algebra $\mathscr{P}$ is a differential graded algebra $\mathscr{P}$, with a Lie algebra homomorphism $\theta: \mathfrak{g} \rightarrow \operatorname{Der}(\mathscr{P})^{0}$ and a linear map $i: \mathfrak{g} \rightarrow$ $\operatorname{Der}(\mathscr{P})^{-1}$, such that the following relationships hold for all $x, y \in \mathfrak{g}$ :

(1) $i([x, y])=\theta(x) i(y)-i(x) \theta(y)$, and

(2) $\theta(x)=\delta i(x)+i(x) \delta$, where $\delta$ is the differential of $\mathscr{P}$. The second relation implies that for all $x \in \mathfrak{g}, \theta(x)$ commutes with $\delta$.

This is equivalent to the following definition. A $\mathfrak{g}$-differential graded algebra is a differential graded algebra $\mathscr{P}$, with a Lie algebra homomorphism $\theta: \mathfrak{g} \rightarrow$ $C(\delta)^{0}$, where $C(\delta)^{0}$ is the degree zero Lie subalgebra of the centralizer of $\delta$ in the graded Lie algebra $\operatorname{Der}(\mathscr{P}$ ) (thus, for each $x \in \mathfrak{g}, \theta(x)$ is a chain map of $\mathscr{P}$ to $\mathscr{P})$, such that for all $x \in \mathfrak{g}$ there is a contracting homotopy, denoted $i(x)$, for $\theta(x)$ and the relation $i([x, y])=\theta(x) i(y)-i(x) \theta(y)$ holds for all $x, y \in \mathfrak{g}$. The canonical example of this type of structure is the differential graded algebra of forms $\Omega^{\bullet}(P)$ of a principal fiber bundle $P$, where the differential is the exterior derivative of forms $d_{P}, i(x)$ is the inner product of a form with the fundamental vector field $s(x)$, and $\theta(x)$ is the Lie derivative of the form along $s(x)$.

Another example of a $\mathfrak{g}$-differential graded algebra is the Chevalley-Eilenberg complex, $C(\mathfrak{g})$, of a Lie algebra $\mathfrak{g}$. As a graded algebra it is $\Lambda\left(\mathfrak{g}^{*}\right)$, where $\mathfrak{g}^{*}$ has degree one. The differential $\delta$ is defined as identically zero on $\Lambda^{0}\left(\mathfrak{g}^{*}\right)=k$. For $\eta \in \Lambda^{1}\left(\mathfrak{g}^{*}\right)=\mathfrak{g}^{*}, \delta \eta$ is the element in $\Lambda^{2}\left(\mathfrak{g}^{*}\right)$ defined by $(\delta \eta)(x, y)=$ $-\eta([x, y]) ; \delta$ is then canonically extended to a derivation on $\Lambda\left(\mathfrak{g}^{*}\right)$. It follows from the Jacobi identity that $\delta$ defined in this manner is a differential. The maps $\theta$ and $i$ are defined to be identically zero on $\Lambda^{0}\left(\mathfrak{g}^{*}\right)$, while for $\eta \in \Lambda^{1}\left(\mathfrak{g}^{*}\right)$, $\theta(x) \eta$ is defined to be $\operatorname{ad}^{*}(x) \eta$ and $i(x) \eta$ is defined to be $\eta(x)$. Both are then canonically extended as derivations of degree zero and -1 , respectively, of $\Lambda\left(\mathfrak{g}^{*}\right)$. 
Explicit formulas for these various maps, which will be useful later on, are obtained by introducing a basis for $\mathfrak{g}^{*}$. Let $\left\{\eta_{\alpha}\right\}_{\alpha=1}^{r}$ be a basis for $\mathfrak{g}$, where $\operatorname{dim}(\mathfrak{g})=r$, and let $\left\{\eta^{\alpha}\right\}_{\alpha=1}^{r}$ be the dual basis of $\mathfrak{g}^{*}$. The structure constants $C_{\beta \gamma}^{\alpha} \in k$, of $\mathfrak{g}$, are defined by the equations $\left[\eta_{\beta}, \eta_{\gamma}\right]=C_{\beta \gamma}^{\alpha} \eta_{\alpha}$, where the [, ] is the Lie bracket of $\mathfrak{g}$, and the convention that repeated indices are summed over is being used, as it will be in all that follows. It follows from the properties of the Lie bracket of $\mathfrak{g}$, that $C_{\beta \gamma}^{\alpha}=-C_{\gamma \beta}^{\alpha}$ and $C_{\beta \beta}^{\alpha}=0$ and from the Jacobi identity it follows that $C_{\alpha \delta}^{\varepsilon} C_{\beta \gamma}^{\delta}+C_{\beta \delta}^{\varepsilon} C_{\gamma \alpha}^{\delta}+C_{\gamma \delta}^{\varepsilon} C_{\alpha \beta}^{\delta}=0$. Using the structure constants of $\mathfrak{g}$ and the above two bases, the explicit formulas for $\delta, \theta$, and $i$ are the following: $\delta\left(\eta^{\alpha}\right)=-\frac{1}{2} C_{\beta \gamma}^{\alpha} \eta^{\gamma} \wedge \eta^{\beta}, \theta\left(\eta_{\beta}\right) \eta^{\alpha}=-C_{\beta \gamma}^{\alpha} \eta^{\gamma}$, and $i\left(\eta_{\beta}\right)\left(\eta^{\alpha}\right)=\delta_{\beta}^{\alpha}$. Note that $\delta^{2}=0$ is equivalent to the relation $C_{\beta \gamma}^{\alpha} C_{\sigma \tau}^{\beta} \eta^{\tau} \wedge \eta^{\sigma} \wedge \eta^{\gamma}=0$.

Finally, given a $\mathfrak{g}$-differential graded algebra $\mathscr{P}$, then, as Cartan defines it in [4], an algebraic connection is a map $A: \mathfrak{g}^{*} \rightarrow \mathscr{P} 1$, which satisfies the relations $i(x) A(\eta)=\eta(x)$ and $\theta(x) A(\eta)=A\left(\operatorname{ad}^{*}(x) \eta\right)$, for all $x \in \mathfrak{g}$ and $\eta \in \mathfrak{g}^{*}$. In the setting of a principal fiber bundle $P$, with $\mathscr{P}=\Omega^{\bullet}(P)$, this is equivalent to the many other definitions of a connection for $P$. The importance of an algebraic connection is that it extends canonically to a graded algebra homomorphism (also denoted $A$ ), $A: \Lambda\left(\mathfrak{g}^{*}\right) \rightarrow \mathscr{P}$, so that $A: \Lambda^{n}\left(\mathfrak{g}^{*}\right) \rightarrow \mathscr{P}^{n}$. In general it is not true that $d A(\eta)=A(\delta \eta)$. The difference, $d A(\eta)-A(\delta \eta) \in \mathscr{P}^{2}$ is the algebraic definition of the curvature of the connection $A$.

3.6. The Weil algebra $W(\mathfrak{g})$. The Weil algebra $W(\mathfrak{g})$, associated to a Lie algebra $\mathfrak{g}$, is the universal $\mathfrak{g}$-differential graded algebra, with a connection $\imath: \mathfrak{g}^{*} \rightarrow W(\mathfrak{g})^{1}$. Thus, given a $\mathfrak{g}$-differential graded algebra $\mathscr{P}$, with a connection $A$, there exists a degree zero $\mathfrak{g}$-differential graded algebra homomorphism, $(A, F): W(\mathfrak{g}) \rightarrow \mathscr{P}$, such that $(A, F) \circ \mathfrak{l}=A$. Furthermore, $W(\mathfrak{g})$ is acyclic.

The underlying graded algebra of the Weil algebra $W(\mathfrak{g})$ is the graded algebra $\Lambda\left(\mathfrak{g}^{*} \oplus \sigma \mathfrak{g}^{*}\right)$ where as before $\operatorname{deg}\left(\mathfrak{g}^{*}\right)=1$. On the one hand, the Koszul differential $\partial$ turns this algebra into a universal differential graded algebra but it does not take into account the $\mathfrak{g}$ structure. On the other hand, given a connection $A: \mathfrak{g}^{*} \rightarrow \mathscr{P}$, there is a unique extension to a graded algebra homomorphism of $\Lambda\left(\mathfrak{g}^{*}\right)$ into $\mathscr{P}$, but it does not, necessarily, commute with the differential $\delta$ of the Chevalley-Eilenberg complex, the canonical $\mathfrak{g}$-differential graded algebra. The differential of the Weil algebra combines the differential of $K(\mathfrak{g})$ and the differential of the Chevalley-Eilenberg complex $C(\mathfrak{g})$ so that $A$ commutes with the resulting differential and the acyclicity of $K(\mathfrak{g})$ is preserved. The linear map $i$ of $C(\mathfrak{g})$ is extended to $S\left(\sigma \mathfrak{g}^{*}\right)$ by defining it to be identically zero. The Lie algebra homomorphism $\theta$ is extended to $\operatorname{Der}\left(S\left(\sigma \mathfrak{g}^{*}\right)\right)$ by defining $\theta(x)(\sigma \eta)=\sigma(\theta(x) \eta)$ and then extending it to all of $S\left(\sigma \mathfrak{g}^{*}\right)$. The differential $\delta$, when it is extended to $S\left(\sigma \mathfrak{g}^{*}\right)$, must satisfy the relation $\theta(x)(\sigma \eta)=i(x) \delta(\sigma \eta)$ and be a degree one homomorphism, i.e. since $S^{n}\left(\sigma \mathfrak{g}^{*}\right) \subset \Lambda^{2 n}\left(\mathfrak{g}^{*} \oplus \sigma \mathfrak{g}^{*}\right), \delta: S^{n}\left(\sigma \mathfrak{g}^{*}\right) \rightarrow \Lambda^{2 n+1}\left(\mathfrak{g}^{*} \oplus \sigma \mathfrak{g}^{*}\right)$ where the latter is isomorphic to $\bigoplus_{p+2 q=2 n+1}\left(E^{p}\left(\mathfrak{g}^{*}\right) \otimes S^{q}\left(\sigma \mathfrak{g}^{*}\right)\right)$. Clearly, these relations imply that $\delta: S^{n}\left(\sigma \mathfrak{g}^{*}\right) \rightarrow E^{1}\left(\mathfrak{g}^{*}\right) \otimes S^{n}\left(\sigma \mathfrak{g}^{*}\right)$. Thus, if $\delta$ is defined on $\sigma \eta \in S^{1}\left(\sigma \mathfrak{g}^{*}\right)$ by $\delta(\sigma \eta)=\eta^{\alpha} \otimes \theta\left(\eta_{\alpha}\right)(\sigma \eta)$, it follows that $i(x) \delta(\sigma \eta)=\eta^{\alpha}(x) \cdot \theta\left(\eta_{\alpha}\right)(\sigma \eta)=$ $\theta(x)(\sigma \eta)$, for $x \in \mathfrak{g}$, as required. Again, $\delta$ is canonically extended, as a derivation, to all of $S\left(\sigma \mathfrak{g}^{*}\right)$ and it follows from the definition that the extension is a differential. This transfers the $\mathfrak{g}$ structure to $S\left(\sigma \mathfrak{g}^{*}\right)$. Written out in detail, 
using a basis of $\mathfrak{g}^{*}$ and the structure constants of $\mathfrak{g}$, the formula for $\delta$ is $\delta\left(\sigma \eta^{\alpha}\right)=C_{\beta \gamma}^{\alpha} \eta^{\gamma} \wedge \sigma \eta^{\beta}$. This defines the differential $\delta$ on all of $\Lambda\left(\mathfrak{g}^{*} \oplus \sigma \mathfrak{g}^{*}\right)$.

The differential $d$ of $W(\mathfrak{g})$ is $d=\partial+(-1)^{q} \delta$, on $E\left(\mathfrak{g}^{*}\right) \otimes S^{q}\left(\sigma \mathfrak{g}^{*}\right)$, where $\partial$ is the differential of $K(\mathfrak{g})$ and $\delta$ is the extended Chevalley-Eilenberg differential. The important result is that $W(\mathfrak{g})$ is acyclic with respect to $d$.

As for $K(\mathfrak{g})$, it will be useful to express the differentials $\delta, \partial$, and $d$, and the homomorphism $\theta$ in terms of a dual basis of $\mathfrak{g}^{*}$ and the structure constants of $\mathfrak{g}$. They are as follows:

$$
\begin{gathered}
\delta\left(\eta^{\alpha}\right)=-\frac{1}{2} C_{\beta \gamma}^{\alpha} \eta^{\gamma} \wedge \eta^{\beta}, \\
\delta\left(\sigma \eta^{\alpha}\right)=C_{\beta \gamma}^{\alpha} \eta^{\gamma} \wedge \sigma \eta^{\beta}, \\
\partial\left(\eta^{\alpha}\right)=\sigma \eta^{\alpha}, \\
\partial\left(\sigma \eta^{\alpha}\right)=0, \\
d\left(\eta^{\alpha}\right)=\sigma \eta^{\alpha}-\frac{1}{2} C_{\beta \gamma}^{\alpha} \eta^{\gamma} \wedge \eta^{\beta}, \\
d\left(\sigma \eta^{\alpha}\right)=-C_{\beta \gamma}^{\alpha} \eta^{\gamma} \wedge \sigma \eta^{\beta}, \\
\theta\left(\eta_{\beta}\right)\left(\eta^{\alpha}\right)=\frac{1}{2} C_{\beta \gamma}^{\alpha} \eta^{\gamma},
\end{gathered}
$$

and

$$
\theta\left(\eta_{\beta}\right)\left(\sigma \eta^{\alpha}\right)=-\frac{1}{2} C_{\beta \gamma}^{\alpha} \sigma \eta^{\gamma}
$$

Finally, given a $\mathfrak{g}$-differential graded algebra $\mathscr{P}$ and a connection $A: \mathfrak{g}^{*} \rightarrow \mathscr{P}$, the map $(A, F): W(\mathfrak{g}) \rightarrow \mathscr{P}$ is defined on the generators of $W(\mathfrak{g})$, as follows: $(A, F)(\eta)=A(\eta)$, and $(A, F)(\sigma \eta)=d A(\eta)-A(\delta \eta)$. Checking the definitions shows that $(A, F)$ is a chain map with respect to the total differential $d$ of $W(\mathfrak{g})$.

\section{THE LOCAL DESCRIPTION OF THE OPERATORS}

The purpose of this section is to describe the operators $\mathscr{L}$ over open sets $U$ of $M$. Since the typical $\mathscr{L}$ is a finite sum of the homogeneous operators $L_{m}$, it suffices to understand the local description of the homogeneous operators $L_{m}$. In what follows the subscript $m$ will be dropped, thus an operator $L$ is to be understood as homogeneous of degree $m$, unless stated otherwise.

Over an open set $U \subset M$, the sections of the bundles are identified as $\left.\Gamma($ ad $P)\right|_{U} \simeq \Omega^{0}(U, \mathfrak{g})$, and $\left.\Omega^{1}(M$, ad $P)\right|_{U} \simeq \Omega^{1}(U, \mathfrak{g})$. Using a basis for the Lie algebra $\mathfrak{g}$, a typical element $f \in \Omega^{0}(U, \mathfrak{g})$ is of the form $f^{\alpha}(\mathbf{x}) \eta_{\alpha}$, while a typical element $\omega \in \Omega^{1}(U, \mathfrak{g})$ is of the form $\omega_{k}^{\alpha}(\mathbf{x}) d x^{k} \otimes \eta_{\alpha}$, where $f^{\alpha}(\mathbf{x})$ and $\omega_{i}^{\alpha}(\mathbf{x})$ are in $C^{\infty}(U)$ with $\mathbf{x} \in U$. In what follows the variable $\mathbf{x}$ will be dropped in the function coefficients of the sections. Thus $f^{\alpha}$ and $\omega_{k}^{\alpha}$ are to be understood as functions depending on $\mathbf{x}$. It follows that locally, an operator $L$ evaluated at $\tilde{f}_{1}, \ldots, \tilde{f}_{p} \in \Gamma(\operatorname{ad} P), \tilde{\omega} \in \Omega^{1}(M$, ad $P)$ is

$$
\left.L\left(\tilde{f}_{1}, \ldots, \tilde{f}_{p}, \tilde{\omega}\right)\right|_{U}=L(\mathbf{x}) D\left(f_{1}, \ldots, f_{p}\right) \cdot \mathscr{D}(\omega) d \mathbf{x}^{I},
$$

where

(1) Chern's convention is being used for the argument $\tilde{\omega}$ and $\omega$,

(2) $f_{i}=\left.\tilde{f}_{i}\right|_{U}$

(3) $\omega=\left.\tilde{\omega}\right|_{U}$ 
(4) $L(\mathbf{x}) \in C^{\infty}(U)$,

(5) $I=\left(i_{1}, \ldots, i_{q}\right)$ with $i_{k} \in(1, \ldots, n), n$ is the dimension of $M$,

(6) $D: \Omega^{0}(U, \mathfrak{g})^{p} \rightarrow C^{\infty}(U)$ is a differential operator from $\Omega^{0}(U, \mathfrak{g})^{p}$ to $C^{\infty}(U)$, and

(7) $\mathscr{D}: \Omega^{1}(U, \mathfrak{g})^{m} \rightarrow C^{\infty}(U)$ is a differential operator from $\Omega^{1}(U, \mathfrak{g})^{m}$ to $C^{\infty}(U)$. The set of operators $L$ will be denoted by $S_{U}^{\bullet, \bullet}$, to emphasize that they are being described over an open set $U \subset M$.

The first step in a fuller description of $L \in S_{U}^{\bullet}, \bullet$ is a better understanding of $D$ and $\mathscr{D}$. The objective is to express $D$ as a product of differential operators from $\Omega^{0}(U, \mathfrak{g})$ to $C^{\infty}(U)$, i.e. $D=D^{1} \wedge \cdots \wedge D^{p}$, where $D^{i}: \Omega^{0}(U, \mathfrak{g}) \rightarrow$ $C^{\infty}(U)$, and to express $\mathscr{D}$ as a product of differential operators from $\Omega^{1}(U, \mathfrak{g})$ to $C^{\infty}(U)$, i.e. $\mathscr{D}=\mathscr{D}^{1} \wedge \cdots \wedge \mathscr{D}^{m}$, where $\mathscr{D}^{i}: \Omega^{1}(U, \mathfrak{g}) \rightarrow C^{\infty}(U)$. Note that $D^{i}$ has total degree one, since $D^{i} \in S_{U}^{1,0}$; while $\mathscr{D}^{i}$ has total degree zero, since $\mathscr{D}^{i} \in S_{U}^{0,0}$. These degrees define the graded product $\wedge$.

The operator $L$ can now be described much more precisely, at the cost of introducing indices. A typical operator, $D: \Omega^{0}(U, \mathfrak{g}) \rightarrow C^{\infty}(U)$, is described by

$$
D\left(f^{\beta} \eta_{\beta}\right)=\partial_{\mu} \otimes \eta^{\alpha}\left(f^{\beta} \eta_{\beta}\right)=(-1)^{\operatorname{deg}\left(\eta^{\alpha}\right) \cdot \operatorname{deg}\left(f^{\beta}\right)} \partial_{\mu} f^{\beta} \eta^{\alpha}\left(\eta_{\beta}\right)=\partial_{\mu} f^{\alpha},
$$

for some multi-index $\mu$ and Lie algebra index $\alpha$. The symbol $\partial_{\mu}$ is shorthand notation for the partial derivative $\partial_{\mu}=\partial^{|\mu|} / \partial x^{\mu_{1}} \cdots \partial x^{\mu_{k}}$ with respect to the multi-index $\mu$, where $|\mu|=k$ is the length of $\mu$, the number of entries in $\mu$, and each $\mu_{i} \in(1, \ldots, n)$. Note that when $|\mu|=0$, then $\mu$ has no entries. In this case it will be written as $\partial_{0}$ and defined by $\partial_{0} f^{\alpha}=f^{\alpha}$. Thus, in general, $D$ will select a "coefficient" $f^{\alpha}$ of $f^{\beta} \eta_{\beta}$ and differentiate it with respect to the multi-index $\mu$.

On the other hand, a typical operator, $\mathscr{D}: \Omega^{1}(U, \mathfrak{g}) \rightarrow C^{\infty}(U)$, is determined by

$$
\begin{aligned}
\mathscr{D}\left(\omega_{k}^{\alpha} d x^{k} \otimes \eta_{\alpha}\right) & =\partial_{\nu} \otimes \frac{\partial}{\partial x^{j}} \otimes \eta^{\beta}\left(\omega_{k}^{\alpha} d x^{k} \otimes \eta_{\alpha}\right) \\
& =(-1)^{1 \cdot 1} \partial_{\nu}\left(\omega_{k}^{\alpha}\right) \frac{\partial}{\partial x_{j}}\left(d x^{k}\right) \eta^{\beta}\left(\eta_{\alpha}\right)=-\partial_{\nu} \omega_{j}^{\beta}
\end{aligned}
$$

for some multi-index $\nu$, Lie algebra index $\beta$, and one-form index $j$. Again, $\partial_{\nu}$ is a partial derivative with respect to the multi-index $\nu$ with the same conventions holding for $\nu$ as for $\mu$. Thus, $\mathscr{D}$ will select a "coefficient" $\omega_{j}^{\beta}$ of $\omega_{k}^{\alpha} d x^{k} \otimes \eta_{\alpha}$ and differentiate it with respect to the multi-index $\nu$.

This information can be codified with the following notation. If $D_{\mu}^{\alpha}$ and $\mathscr{D}_{\nu, j}^{\beta}$ are defined as $D_{\mu}^{\alpha}\left(f^{\beta} \eta_{\beta}\right)=\partial_{\mu} f^{\alpha}$ and $\mathscr{D}_{\nu, j}^{\beta}\left(\omega_{k}^{\alpha} d x^{k} \otimes \eta_{\alpha}\right)=-\partial_{\nu} \omega_{j}^{\beta}$, then $L\left(\tilde{f}_{1}, \ldots, \tilde{f}_{p}, \tilde{\omega}\right)$ in local coordinates is described by

$$
L_{A B I}^{M N J}(\mathbf{x}) D_{\mu_{1}}^{\alpha_{1}} \wedge \cdots \wedge D_{\mu_{p}}^{\alpha_{p}}\left(f_{1}, \ldots, f_{p}\right) \cdot \mathscr{D}_{\nu_{1}, j_{1}}^{\beta_{1}} \wedge \cdots \wedge \mathscr{D}_{\nu_{m}, j_{m}}^{\beta_{m}}(\omega) d \mathbf{x}^{I},
$$

where, as always, the summation convention and Chern's convention are being used. The indices on the function coefficient $L_{A B}^{M N J}(\mathbf{x})$ are

(1) $M=\left(\mu_{1}, \ldots, \mu_{p}\right)$, each $\mu_{i}$ a multi-index,

(2) $A=\left(\alpha_{1}, \ldots, \alpha_{p}\right)$, each $\alpha_{i}$ a Lie algebra index,

(3) $N=\left(\nu_{1}, \ldots, \nu_{m}\right)$, each $\nu_{i}$ a multi-index, 
(4) $J=\left(j_{1}, \ldots, j_{m}\right)$, each $j_{i} \in(1, \ldots, n)$,

(5) $B=\left(\beta_{1}, \ldots, \beta_{m}\right)$, each $\beta_{i}$ a Lie algebra index,

(6) $I=\left(i_{1}, \ldots, i_{q}\right)$, each $i_{k} \in(1, \ldots, n)$.

In the calculations which occur below, the following notation will be adopted. The local representation of $\left.L\right|_{U}$ will be written as

$$
\left.L\right|_{U}=L_{A B}^{M N J}{ }_{I}(\mathbf{x}) D_{M}^{A} \mathscr{D}_{N, J}^{B} d \mathbf{x}^{I},
$$

so that

$$
\left(\left.L\right|_{U}\right)\left(f_{1}, \ldots, f_{p}, \omega\right)=L_{A B}^{M N J}(\mathbf{x}) D_{M}^{A}\left(f_{1}, \ldots, f_{p}\right) \cdot \mathscr{D}_{N, J}^{B}(\omega) d \mathbf{x}^{I},
$$

where

$$
D_{M}^{A}=D_{\mu_{1}}^{\alpha_{1}} \wedge \cdots \wedge D_{\mu_{p}}^{\alpha_{p}}, \quad \text { and } \quad \mathscr{D}_{N, J}^{B}=\mathscr{D}_{\nu_{1}, j_{1}}^{\beta_{1}} \wedge \cdots \wedge \mathscr{D}_{\nu_{m}, j_{m}}^{\beta_{m}} .
$$

Note that $M$ and $A$ are paired as multi-indices so that under odd permutations of pairs $\left(\mu_{i}, \alpha_{i}\right), L_{A B}^{M N J}(\mathbf{x})$ changes sign, since the operator $D_{\mu_{1}}^{\alpha_{1}} \wedge \cdots \wedge D_{\mu_{p}}^{\alpha_{p}}$ is skew symmetric. On the other hand, $N, B$ and $J$ are "paired"; but under permutations of triples $\left(\nu_{i}, \beta_{i}, j_{i}\right), L_{A B}^{M N J} I_{I}(\mathbf{x})$ does not change sign, since the operator $\mathscr{D}_{\nu_{1}, j_{1}}^{\beta_{1}} \wedge \cdots \wedge \mathscr{D}_{\nu_{m}, j_{m}}^{\beta_{m}}$ is symmetric. Of course, since $I$ is a multiindex for $d \mathbf{x}^{I}, L_{A B}^{M N J}(\mathbf{x})$ will change sign under odd permutations of $i^{\prime} s \in I$. Furthermore, if $I$ contains two identical entries, so that $d \mathbf{x}^{I}=0$, then the coefficient $L_{A B}^{M N J} I_{I}(\mathbf{x})$ is also 0 and similarly if two pairs $\left(\alpha_{i}, \mu_{i}\right)$ and $\left(\alpha_{j}, \mu_{j}\right)$ are the same.

The following conventions will be used for the lengths of the multi-indices. For $A, B, I, J$, the length is defined, as usual, to be the number of elements in the index, thus, for example, $|A|=p$. This implies that if $L \in S_{U}^{p, \bullet}$ then $|A|$ must be equal to $p$, while if $L \in S_{U}^{\bullet, q}$, then $|I|$ must be equal to $q$, and if $L$ is homogeneous of degree $m$, then $|B|=|J|=m$. For the multi-indices $M, N$, the length is defined to be the sum of the lengths of the multi-indices they contain. Thus, for example, $|M|=\left|\mu_{1}\right|+\cdots+\left|\mu_{p}\right|$ and it should be noted that in general there is no relation between $|M|$ and $p$. The length of $|M|$ is determined by the sum of the lengths of the $p$ multi-indices but may be less than, equal to, or greater than $p$. Furthermore, even if $|M|=0$, so that each $\left|\mu_{i}\right|=0, A$ may still be a nonempty multi-index. Conforming to the previous convention, in this case, the subscript $M$ on $D_{M}^{A}$ will be written as 0 so that $D_{0}^{A}=D_{0}^{\alpha_{1}} \wedge \cdots \wedge D_{0}^{\alpha_{p}}$. Of course, all this holds for $N$ as well. Thus $|N|$ may be greater than, equal to, or less than $m$, and if each $\left|\nu_{i}\right|=0$, neither $B$ nor $J$ need be empty. In the latter case, as for $M$, the subscript $N$ on $\mathscr{D}_{N, J}^{B}$ will be written as 0 , so that $\mathscr{D}_{0, J}^{B}=\mathscr{D}_{0, j_{1}}^{\beta_{1}} \wedge \cdots \wedge \mathscr{D}_{0, j_{m}}^{\beta_{m}}$. It should be noted that the total order of $L$ as a differential operator is $|M|+|N|$. In the special case where $|I|=0$, by convention $d \mathbf{x}^{0}=1$. This occurs in the case that an operator $L$ is in $S_{U}^{p, 0}$. Furthermore by convention if $A$ is empty as a multi-index, then $A$ will be denoted by 0 and $D_{0}^{0}=1$ and similarly if $B$ is empty as a multi-index, then $\mathscr{D}_{0,0}^{0}=1$.

\section{INVARIANCE CALCULATIONS}

For an operator $L$ to be invariantly defined on $M$, it must in principal be definable without reference to a local coordinate system. In other words, 
the operator $L$ must not depend upon its coordinate description. If $\phi: U \rightarrow$ $V \subset \mathbb{R}^{n}$ is a local coordinate system, then for all $\Phi \in \operatorname{Diff}\left(\mathbb{R}^{n}\right)$, the group of diffeomorphisms of $\mathbb{R}^{n}$, the composition

$$
\Phi \circ \phi: U \rightarrow \bar{V}=\Phi(V) \subset \mathbb{R}^{n}
$$

is also a local coordinate system. In fact $\Phi$ does not have to be in the full diffeomorphism group, it suffices that $\Phi$ be a local diffeomorphism, i.e. $\Phi$ maps the open set $V \subset \mathbb{R}^{n}$ diffeomorphically to some other open set $\bar{V} \subset \mathbb{R}^{n}$. This is the reason for considering the pseudo-group of local diffeomorphisms of $\mathbb{R}^{n}$, which will be denoted by $\operatorname{Diff}_{\text {loc }}\left(\mathbb{R}^{n}\right)$. By definition $\Phi \in \operatorname{Diff}$ loc $\left(\mathbb{R}^{n}\right)$ means that there exist open sets $V, \bar{V}$ of $\mathbb{R}^{n}$ and that $\Phi$ is a diffeomorphism of $V$ and $\bar{V}$, i.e. Diff loc $\left(\mathbb{R}^{n}\right)$ is a category where the objects are open sets in $\mathbb{R}^{n}$ and the morphisms are diffeomorphisms. Thus the operators $L$ which make the following diagram commute for all $\Phi \in \operatorname{Diff}_{\text {loc }}\left(\mathbb{R}^{n}\right)$ do not depend upon the coordinate system $(\phi, U)$,

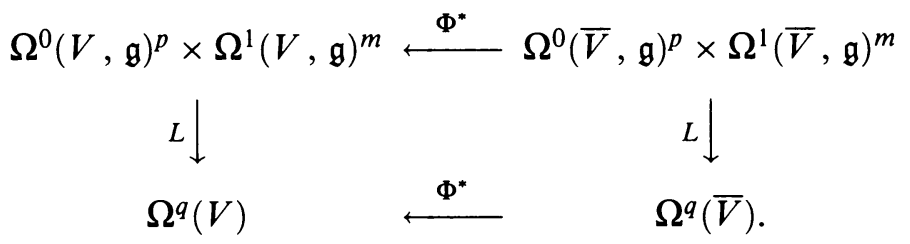

To simplify the notation the coordinate system $(\phi, U)$ has been dropped and the operators $L$ are identified with their description over the open sets $V$ and $\bar{V}$ of $\mathbb{R}^{n}$. This is equivalent to finding the $L$ such that

$$
\begin{aligned}
& \Phi^{*}\left(\bar{L}_{A B I I}^{M N J}(\overline{\mathbf{x}}) \bar{D}_{M}^{A} \overline{\mathscr{D}}_{N, J}^{B}\left(\bar{f}_{1}, \ldots, \bar{f}_{p}, \bar{\omega}\right) d \overline{\mathbf{x}}^{I}\right) \\
& \quad=L_{A B}^{M N J}{ }_{I}(\mathbf{x}) D_{M}^{A} \mathscr{D}_{N, J}^{B}\left(\Phi^{*}\left(\bar{f}_{1}\right), \ldots, \Phi^{*}\left(\bar{f}_{p}\right), \Phi^{*}(\bar{\omega})\right) d \mathbf{x}^{I},
\end{aligned}
$$

or in shorthand,

$$
\Phi^{*} \circ\left(\bar{L}_{A B}^{M N J}(\overline{\mathbf{x}}) \bar{D}_{M}^{A} \overline{\mathscr{D}}_{N,{ }_{J}}^{B} d \overline{\mathbf{x}}^{I}\right)=\left(L_{A B}^{M N J}(\mathbf{x}) D_{M}^{A} \mathscr{D}_{N, J}^{B} d \mathbf{x}^{I}\right) \circ \Phi^{*},
$$

for all $\Phi \in \operatorname{Diff}_{\text {loc }}\left(\mathbb{R}^{n}\right)$, where a bar means that the operator $L$ and the various arguments of $L$ are expressed in the coordinates of $\bar{V}$. The operators in $S_{U}^{\bullet, \bullet}$ which are invariant under all of $\operatorname{Diff}_{\mathrm{loc}}\left(\mathbb{R}^{n}\right)$ will be denoted by $\left(S_{U}^{\bullet, \bullet}\right)^{\operatorname{Difloc}}\left(\mathbb{R}^{n}\right)$. Also, notice that by their definition the multi-indices $M, N$, and $J$ are contravariant ( $j \in J$ being dual to a covariant index), while the multi-index $I$ is covariant.

To find the necessary and sufficient conditions on the $L$ 's to make them invariant under $\operatorname{Diff}_{\text {loc }}\left(\mathbb{R}^{n}\right)$, the action of successively more complicated elements of Diff loc $\left(\mathbb{R}^{n}\right)$ will be examined. The conditions for invariance of the operators $L$ will proceed in the following order:

(1) translation,

(2) dilation, and

(3) invariance under the action of the orthogonal group $O(n)$. In $\S 7$, it will be shown that the operators which are invariant under the above three types of elements of $\operatorname{Diff}$ loc $\left(\mathbb{R}^{n}\right)$, are invariant under all of $\operatorname{Diff}_{\text {loc }}\left(\mathbb{R}^{n}\right)$.

In what follows, the domain of $\Phi \in \operatorname{Diff}_{\text {loc }}\left(\mathbb{R}^{n}\right)$ will always be $V$ and the range will always be $\bar{V}$, so that $\Phi^{*}: \Omega^{q}(\bar{V}) \rightarrow \Omega^{q}(V)$ and $\Phi^{*}: \mathscr{X}(\bar{V}) \rightarrow \mathscr{X}(V)$ 
where $\mathscr{X}(V)$ is the set of vector fields on $V$ (vector fields can be pulled back since $\Phi$ is a diffeomorphism). All other notation will also follow this convention; thus $\bar{D}_{\mu}^{\alpha}$ will be expressed in the $\overline{\mathbf{x}}$ coordinates of $\bar{V}$, and $D_{\mu}^{\alpha}$ in the $\mathbf{x}$ coordinates of $V$.

Proposition 5.1 (Translations $\overline{\mathbf{x}}=\Phi(\mathbf{x})=\mathbf{x}+\lambda, \lambda \in \mathbb{R}^{n}$ ). The action of $a$ translation $\Phi$ on an operator $L$ is as follows:

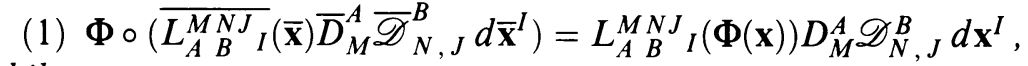
while

(2) $\left(L_{A B}^{M N J}{ }_{I} D_{M}^{A} \mathscr{D}_{N, J}^{B} d \mathbf{x}^{I}\right) \circ \Phi^{*}=L_{A B}^{M N J}{ }_{I}(\mathbf{x}) D_{M}^{A} \mathscr{D}_{N, J}^{B} d \mathbf{x}^{I}$.

Thus, if $L$ is to be invariant under the action of all translations $\Phi$, then it follows that $L_{A B}^{M N J}{ }_{I}(\mathbf{x}+\lambda)=L_{A B}^{M N J}{ }_{I}(\mathbf{x})$ for all $\lambda$. Therefore $L_{A B}^{M N J}{ }_{I}(\mathbf{x})$ is a constant depending only on the multi-indices $M, N, J, A, B$, and $I$. This implies that the operators $D_{M}^{A} \mathscr{D}_{N, J}^{B} d \mathbf{x}^{I}$ form a basis of the vector space of operators in $S_{U}^{p, q}$ which are invariant under translations.

Proof. Since the proof of this proposition is a lengthy but easy calculation involving the properties of covariance and contravariance under pullback by a diffeomorphism and the definitions of the various operators and their arguments, it is not included here.

Proposition 5.2 (Dilations $\bar{x}=\Phi(\mathbf{x})=\lambda \mathbf{x}, \lambda \in \mathbb{R}$ ). The action of the dilations decomposes the space of operators $L$ which are invariant under translation into weight spaces depending on the lengths of the multi-indices $M, N, J, I$. Specifically, the action of a dilation $\Phi$ on an operator $L$ is

$$
\Phi^{*} \circ\left(\bar{L}_{A B}^{M N J}{ }_{I} \bar{D}_{M}^{A} \overline{\mathscr{D}}_{N, J}^{B} d \overline{\mathbf{x}}^{I}\right)=\lambda^{-|M|-|N|+|I|} L_{A B}^{M N J}, D_{M}^{A} \mathscr{D}_{N, J}^{B} d \mathbf{x}^{I}
$$

while

$$
\left(L_{A B}^{M N J}{ }_{I} D_{M}^{A} \mathscr{D}_{N, J}^{B} d \mathbf{x}^{I}\right) \circ \Phi^{*}=\lambda^{|J|} L_{A B}^{M N J}{ }_{I} D_{M}^{A} \mathscr{D}_{N, J}^{B} d \mathbf{x}^{I} .
$$

Thus, for $L$ to be invariant under the action of dilations, the multi-indices must satisfy the relation $-|M|-|N|+|I|=|J|$ or, equivalently, $|M|+|N|+|J|=$ $|I|$. This is the first constraint on the multi-indices $M, N, J$, and $I$. It implies that if $L \in S_{U}^{p, q}$, then the total order of the differentiation must be less than or equal to $q$, since $|I|=q$ and $|M|+|N| \leq|I|$. Furthermore, the homogeneous degree of $L$ must also be less than or equal to $q$, since $|J| \leq|I|$. Note that if $|I|=0$, then $|J|=|M|=|N|=0$. This implies that any operator in $S_{U}^{\bullet, 0}$ invariant under both translation and dilation must be homogeneous of degree zero and can only have order zero as a differential operator. Hence, the only operators in $S_{U}^{0,0}$ invariant under both translation and dilation are the constant functions.

Proof. The proof of this proposition is completely similar to the proof of Proposition 5.1 and, again, is not included here.

Proposition 5.3 (Invariance under the orthogonal group $O(n)$ ). A necessary and sufficient condition for an operator $L_{A B}^{M N J}{ }_{I} D_{M}^{A} \mathscr{D}_{N, J}^{B} d \mathbf{x}^{I}$ which is already invariant under translations and dilations to be invariant under the action of $O(n)$ is that it be completely contracted in the indices $M, N, J$, and $I$. This implies that $0 \leq|\mu|,|\nu| \leq 1$, for all $\mu \in M$ and $\nu \in N$. 
Proof. The typical operator of homogeneous degree $m$ in $S_{U}^{p, q}$ invariant under translation and dilation is of the form $L_{A B}^{M N J}{ }_{I} D_{M}^{A} \mathscr{D}_{N, J}^{B} d \mathbf{x}^{I}$ where $L_{A B}^{M N J}$ is a constant, $|A|=p,|B|=|J|=m$, and $|I|=q$. It follows from Proposition 5.2 that $|M|+|N|+|J|=|I|$, and hence, $|M|+|N|=q-m$. Therefore, there are the following relations among the multi-indices $\mu \in M$ and $\nu \in N: 0 \leq|\mu| \leq q-m$ for all $\mu, 0 \leq|\nu| \leq q-m$ for all $\nu$, and $\left|\mu_{1}\right|+\cdots+\left|\mu_{p}\right|+\left|\nu_{1}\right|+\cdots+\left|\nu_{m}\right|=q-m$.

Weyl's first main theorem $[15,17]$ on the invariants of $O(n)$ states that all scalars, depending on covariant $n$-vectors $\xi^{i}$ and contravariant $n$-vectors $x_{j}$, invariant under the action of $O(n)$ on $\xi^{i}$ and $x_{j}$, are polynomials in $\xi^{i}\left(x_{j}\right)$. This implies that the only invariant tensor products of covariant and contravariant vectors are completely contracted; in other words, the covariant and contravariant indices are paired and summed over. Hence, the operator $L_{A B}^{M N J}{ }_{I} D_{M}^{A} \mathscr{D}_{N, J}^{B} d \mathbf{x}^{I}$ is of the form $k_{A B} D_{M}^{A} \mathscr{D}_{N, J}^{B} d \mathbf{x}^{\left(i_{1}, \ldots, i_{q-m}, i_{q-m+1}, \ldots, i_{q}\right)}$, where the first $q-m$ entries of $I$ are distributed through $M$ and $N$ in some manner and $k_{A B}$ is a constant depending on the multi-indices $A$ and $B$. Since $|J|=m$ and $|j|=1$ for all $j \in J$, the last $m$ entries of $I$ are paired with the $m$ entries of $J$.

Suppose that $|\mu| \geq 2$ for some $\mu \in M$. On the one hand, since $\mu$ is a multiindex representing a partial derivative, it is symmetric in its entries; but, on the other hand, $I$, being the multi-index of the form degree, is skew symmetric in its entries. Thus, if $|\mu| \geq 2$, then the contracted tensor will have skew symmetric upper indices paired with symmetric lower indices. This implies that the resulting $O(n)$ invariant is the zero invariant. A similar argument holds when $|\nu| \geq 2$ for some $\nu \in N$. Consequently, the only nonzero invariant operators are those where $0 \leq|\mu| \leq 1$ for all $\mu \in M$ and $0 \leq|\nu| \leq 1$ for all $\nu \in N$, i.e. the nonzero invariant operators have partials of at most order 1 . Hence, an invariant operator has the form,

$$
k_{\left(\alpha_{1}, \ldots, \alpha_{p}\right),\left(\beta_{1}, \ldots, \beta_{m}\right)} D_{\left(i_{1}, \ldots, i_{s}, 0, \ldots, 0\right)}^{\left(\alpha_{1}, \ldots, \alpha_{p}\right)} \mathscr{D}_{\left(i_{s+1}, \ldots, i_{l}, 0, \ldots, 0\right),\left(i_{t+1}, \ldots, i_{q}\right)}^{\left(\beta_{1}, \ldots, \beta_{m}\right)} d \mathbf{x}^{\left(i_{1}, \ldots, i_{q}\right)},
$$

where $\left(i_{1}, \ldots, i_{s}\right) \cup\left(i_{s+1}, \ldots, i_{t}\right) \cup\left(i_{t+1}, \ldots, i_{q}\right)=\left(i_{1}, \ldots, i_{q}\right)$ and the zero entries have been gathered at the end of the multi-indices $M$ and $N$, by reordering the pairs $\left(\mu_{i}, \alpha_{i}\right)$ and triples $\left(\nu_{i}, \beta_{i}, j_{i}\right)$, at the cost of a possible sign change due to the reordering of the $\left(\mu_{i}, \alpha_{i}\right)$.

Let $A(n)$ denote the subgroup of $\operatorname{Diff}_{\text {loc }}\left(\mathbb{R}^{n}\right)$ generated by the above three types of diffeomorphisms, then, in what follows, the set of operators which are invariant under the action of $A(n)$ will be denoted by $\left(S_{U}^{\bullet, \bullet}\right)^{A(n)}$. They have as a basis operators of the form

$$
D_{\left(i_{1}, \ldots, i_{s}, 0, \ldots, 0\right)}^{\left(\alpha_{1}, \ldots, \alpha_{p}\right)} \mathscr{D}_{\left(i_{s+1}, \ldots, i_{t}, 0, \ldots, 0\right),\left(i_{t+1}, \ldots, i_{q}\right)}^{\left(\beta_{1}, \ldots, \beta_{m}\right)} d \mathbf{x}^{\left(i_{1}, \ldots, i_{q}\right)} .
$$

\section{FOUR TYPES OF INVARIANT OPERATORS}

Four invariant operators will be described in this section. It will be shown, in $\S 7$, that they generate all of $\left(S_{U}^{\bullet, \bullet}\right)^{\mathrm{Diff}} \mathrm{loc}\left(\mathbb{R}^{n}\right)$ as a graded algebra. As always $\left\{\eta_{\alpha}\right\}$ is a fixed basis for $\mathfrak{g}$ and $\left\{\eta^{\alpha}\right\}$ the corresponding dual basis.

Proposition. The four operators (1) $D_{0}^{\alpha} \in S_{U}^{1,0}$,

(2) $D_{i}^{\alpha} d x^{i} \in S_{U}^{1,1}$,

(3) $\mathscr{D}_{0, i}^{\alpha} d x^{i} \in S_{U}^{0,1}$, and 
(4) $\mathscr{D}_{i, j}^{\alpha} d x^{i} d x^{j} \in S_{U}^{0,2}$ are all invariant, where $\alpha$ is a Lie algebra index.

Proof. Notice the following: (1) the operator $D_{0}^{\alpha}$, evaluated at $f^{\beta} \eta_{\beta}$, produces $D_{0}^{\alpha}\left(f^{\beta} \eta_{\beta}\right)=f^{\alpha}$,

(2) the operator $D_{i}^{\alpha} d x^{i}$, evaluated at $f^{\beta} \eta_{\beta}$, produces

$$
D_{i}^{\alpha} d x^{i}\left(f^{\beta} \eta_{\beta}\right)=\frac{\partial}{\partial x^{i}} f^{\alpha} d x^{i}=d_{\mathbb{R}^{n}}\left(f^{\alpha}\right),
$$

(3) the operator $\mathscr{D}_{0, i}^{\alpha} d x^{i}$, evaluated at $\omega_{k}^{\beta} d x^{k} \otimes \eta_{\beta}$, produces

$$
\mathscr{D}_{0, i}^{\alpha} d x^{i}\left(\omega_{k}^{\beta} d x^{k} \otimes \eta_{\beta}\right)=-\omega_{i}^{\alpha} d x^{i},
$$

and

(4) the operator $\mathscr{D}_{i, j}^{\alpha} d x^{i} d x^{j}$, evaluated at $\omega_{k}^{\beta} d x^{k} \otimes \eta_{\beta}$, produces

$$
\mathscr{D}_{i, j}^{\alpha} d x^{i} d x^{j}\left(\omega_{k}^{\beta} d x^{k} \otimes \eta_{\beta}\right)=-\frac{\partial}{\partial x^{i}} \omega_{j}^{\alpha} d x^{i} d x^{j}=-d_{\mathbb{R}^{n}}\left(\omega_{j}^{\alpha} d x^{j}\right) .
$$

That these four operators are invariant follows from the properties of covariance, contravariance and the elementary fact that diffeomorphisms commute with exterior differentiation. The details are not included here.

Clearly, since $A(n)$ is contained in $\operatorname{Diff}_{\text {loc }}\left(\mathbb{R}^{n}\right)$, it follows that $\left(S_{U}^{\bullet, \bullet}\right)^{\operatorname{Diff}_{\text {loc }}\left(\mathbb{R}^{n}\right)}$ is contained in $\left(S_{U}^{\bullet},\right)^{A(n)}$. Thus the above four operators are contained in $\left(S_{U}^{\bullet, \bullet}\right)^{A(n)}$. Furthermore, since $\operatorname{Diff}_{\text {loc }}\left(\mathbb{R}^{n}\right)$ acts as graded algebra endomorphisms on $S_{U}^{\bullet, \bullet}$, the subalgebra generated by these four operators will be contained in $\left(S_{U}^{\bullet, \bullet}\right)^{\operatorname{Diff}_{\text {loc }}\left(\mathbb{R}^{n}\right)}$. The objective is to show that the subalgebra they generate is, in fact, $\left(S_{U}^{\bullet}, \bullet\right)^{\operatorname{Diff}_{\text {loc }}\left(\mathbb{R}^{n}\right)}$. This will be accomplished, in the next section, by showing that the algebra they generate, here denoted by $\mathscr{A}$, is $\left(S_{U}^{\bullet, \bullet}\right)^{A(n)}$. Therefore there will be the following relationships:

$$
\left(S_{U}^{\bullet, \bullet}\right)^{\operatorname{Diff}_{\text {loc }}\left(\mathbb{R}^{n}\right)} \subset\left(S_{U}^{\bullet, \bullet}\right)^{A(n)}=\mathscr{A} \subset\left(S_{U}^{\bullet, \bullet}\right)^{\operatorname{Diff}_{\mathrm{loc}}\left(\mathbb{R}^{n}\right)},
$$

which, of course, imply equality, i.e. $\left(S^{\bullet}, \bullet\right)^{\operatorname{Diff}}{ }_{\text {loc }}\left(\mathbb{R}^{n}\right)=\left(S_{U}^{\bullet, \bullet}\right)^{A(n)}$. The first step in this process is to examine the vector spaces these four types of operators span in $\left(S_{U}^{\bullet, \bullet}\right)^{A(n)}$.

Consider an arbitrary operator $L \in S_{U}^{1,0}$. What properties must $L$ have to satisfy the criteria of $\S 5$ for invariance under $A(n)$ ? In this case, since $|I|=0$, it follows that $|J|=0$, because $0 \leq|J| \leq|I|$ by $\S 5.2$. This implies that $L$ is homogeneous of degree zero, since $|J|$ is the homogeneous degree of $L$ and thus $|B|=|N|=0$. Furthermore, since $L \in S_{U}^{1,0}$, it follows that $|A|=1$ and that $|M|=0$, since $|M| \leq|I|$. Therefore, $L$ is a linear combination of operators of the form $D_{0}^{\alpha}$, i.e. $L=k_{\alpha} D_{0}^{\alpha}$, for some constants $k_{\alpha} \in k$. This implies that the operators $D_{0}^{\alpha}$ form a basis of $\left(S_{U}^{1,0}\right)^{A(n)}$. But it was shown that these operators are in $\left(S_{U}^{1,0}\right)^{\operatorname{Diff}_{\text {loc }}\left(\mathbb{R}^{n}\right)}$. Hence, there is the following relations between the various vector spaces:

$$
\left(S_{U}^{1,0}\right)^{\operatorname{Diff}_{\text {loc }}\left(\mathbb{R}^{n}\right)} \subset\left(S_{U}^{1,0}\right)^{A(n)}=\left\langle D_{0}^{\alpha}\right\rangle \subset\left(S_{U}^{1,0}\right)^{\operatorname{Diff}}{ }_{\text {loc }}\left(\mathbb{R}^{n}\right),
$$

which imply that as vector spaces they are equal $\left(S_{U}^{1,0}\right)^{\operatorname{Diff}}{ }_{\text {loc }}\left(\mathbb{R}^{n}\right)$ (here \langle\rangle denotes the linear span of the operators $D_{0}^{\alpha}$ ).

Next, consider an operator $L \in S_{U}^{1,1}$ which is homogeneous of degree zero, so that $|J|=|B|=|N|=0$. What properties must $L$ satisfy to be in $\left(S_{U}^{1,1}\right)^{A(n)}$ ? 
First, it follows that $|A|=1$ and $|M|=|I|=1$ and finally, $L$ must be fully contracted. Thus, it is a linear combination of operators of the form $D_{i}^{\alpha} d x^{i}$, i.e. $L=k_{\alpha} D_{i}^{\alpha} d x^{i}$, for some constants $k_{\alpha} \in k$. These were seen to be in $\left(S_{U}^{1,1}\right)^{\text {Diffloc }}\left(\mathbb{R}^{n}\right)$. Hence, the operators $L$ in $\left(S_{U}^{1,1}\right)^{A(n)}$, which have homogeneous degree zero, are spanned as a vector space by $D_{i}^{\alpha} d x^{i}$ and therefore are in $\left(S_{U}^{1,1}\right)^{\operatorname{Diff}}{ }_{\text {loc }}\left(\mathbb{R}^{n}\right)$.

Next, consider an arbitrary operator $L \in S_{U}^{0,1}$. What properties must it satisfy to be in $\left(S_{U}^{0,1}\right)^{A(n)}$ ? Since $L \in S_{U}^{0,1}$, its vertical degree is 0 , so that $|M|=|A|=0$. Because $|N|+|J|=|I|=1$ and $|N| \leq|J|$, it follows that $|J|=1$ and $|N|=0$. This implies that $L$ has homogeneous degree one and, since it must be fully contracted, it is a linear combination of operators of the form $\mathscr{D}_{0, i}^{\alpha} d x^{i}$, i.e. $L=k_{\alpha} D_{0, i}^{\alpha} d x^{i}$, for some constants $k_{\alpha} \in k$. These were seen to be in $\left(S_{U}^{0,1}\right)^{\mathrm{Diff}_{\text {loc }}\left(\mathbb{R}^{n}\right)}$. Hence, there is the following relations between the various vector spaces:

$$
\left(S_{U}^{0,1}\right)^{\mathrm{Diff}_{\mathrm{loc}}\left(\mathbb{R}^{n}\right)} \subset\left(S_{U}^{0,1}\right)^{A(n)}=\left\langle\mathscr{D}_{0, i}^{\alpha} d x^{i}\right\rangle \subset\left(S_{U}^{0,1}\right)^{\mathrm{Diff}_{\mathrm{loc}}\left(\mathbb{R}^{n}\right)},
$$

which imply that as vector spaces they are equal.

Finally, consider an operator $L$ in $S_{U}^{0,2}$. Assume that $L$ has homogeneous degree one. What properties must $L$ satisfy to be in $\left(S_{U}^{0,2}\right)^{A(n)}$ ? As above, since $L$ has vertical degree zero and is assumed to have homogeneous degree one, it follows that $|A|=|M|=0$ and $|J|=1$. Because of the relation $|N|+|J|=|I|=2$, it follows that $|N|=1$. But $L$ must be fully contracted and, therefore, is a linear combination of operators of the form $\mathscr{D}_{i, j}^{\alpha} d x^{i} d x^{j}$, i.e. $L=k_{\alpha} \mathscr{D}_{i, j}^{\alpha} d x^{i} d x^{j}$, for some constants $k_{\alpha} \in k$. These were seen to be in $\left(S_{U}^{0,2}\right)^{\mathrm{Diff}}{ }_{\mathrm{loc}}\left(\mathbb{R}^{n}\right)$. Hence, the operators of homogeneous degree one in $\left(S_{U}^{0,2}\right)^{A(n)}$ are in $\left(S_{U}^{0,2}\right)^{\text {Diffloc }}\left(\mathbb{R}^{n}\right)$ and they have as a basis the operators $\mathscr{D}_{i, j}^{\alpha} d x^{i} d x^{j}$.

Note that in each case, the vector space spanned by these four types of operators is isomorphic to $\mathfrak{g}^{*}$, where the isomorphism is the obvious one, i.e. for each $k_{\alpha} \eta^{\alpha} \in \mathfrak{g}^{*}$ the corresponding operators are

(1) $k_{\alpha} D_{0}^{\alpha}$,

(2) $k_{\alpha} D_{i}^{\alpha} d x^{i}$,

(3) $k_{\alpha} \mathscr{D}_{0, i}^{\alpha} d x^{i}$, and

(4) $k_{\alpha} \mathscr{D}_{i, j}^{\alpha} d x^{i} d x^{j}$ respectively.

Finally this cumbersome notation can be simplified. Note that the above four types of operators do not depend on $n$, the dimension of the manifold $M$, beyond that the sum is over $n$ indices; the only free index is the Lie algebra index $\alpha$. These operators will be renamed as follows.

Since the operator $D_{0}^{\alpha}$, when evaluated at $f^{\beta} \eta_{\beta}$ produces $f^{\alpha}$, the function "coefficient" of $\eta_{\alpha}$, it will be denoted, by an abuse of notation, as $\eta^{\alpha}$ so that $\eta^{\alpha}\left(f^{\beta} \eta_{\beta}\right)=f^{\alpha}$, in analogy to its "dual" nature with respect to $\eta_{\alpha}$, the basis of $\mathfrak{g}$.

The operator $D_{i}^{\alpha} d x^{i}$ produces $d_{\mathbb{R}^{n}} f^{\alpha}$, the exterior derivative of $\eta^{\alpha}$ evaluated at $f^{\beta} \eta_{\beta}$. Notice, by the definition of the differential $\partial$ for the bicomplex $S_{M}^{\bullet, \bullet}$ translated to this local setting, that $\left(\partial \eta^{\alpha}\right)\left(f^{\beta} \eta_{\beta}\right)=d_{\mathbb{R}^{n}} f^{\alpha}=D_{i}^{\alpha} d x^{i}\left(f^{\beta} \eta_{\beta}\right)$. Foreshadowing things to come, the operator $D_{i}^{\alpha} d x^{i}$ will be denoted by $\partial \eta^{\alpha}$, so that $\partial \eta^{\alpha}\left(f^{\beta} \eta_{\beta}\right)=d_{\mathbb{R}^{n}}\left(f^{\alpha}\right)$. 
The operator $\mathscr{D}_{0, i}^{\alpha} d x^{i}$, when evaluated at $\omega_{k}^{\beta} d x^{k} \otimes \eta_{\beta}$, produces $-\omega_{i}^{\alpha} d x^{i}$, and thus is, in the same loose sense, "dual" to the $\eta_{\alpha}$. In this case the "coefficients" are one-forms, since the domain of the operator is $\Omega^{1}(U, \mathfrak{g})$. To distinguish this operator from $\eta^{\alpha}$, but still to capture its "dual" nature, it will be denoted by $\rho^{\alpha} \equiv \mathscr{D}_{0, i}^{\alpha} d x^{i}$, so that $\rho^{\alpha}\left(\omega_{k}^{\beta} d x^{k} \otimes \eta_{\beta}\right)=-\omega_{i}^{\alpha} d x^{i}$.

Finally, the operator $\mathscr{D}_{i, j}^{\beta} d x^{i} d x^{j}$ produces $-d_{\mathbb{R}^{n}}\left(\omega_{i}^{\alpha} d x^{i}\right)$, the exterior derivative of $\rho^{\alpha}$ evaluated at $\omega_{k}^{\beta} d x^{k} \otimes \eta_{\beta}$. Again, by the definition of the differential $\partial$, it follows that

$$
\left(\partial \rho^{\alpha}\right)\left(\omega_{k}^{\beta} d x^{k} \otimes \eta_{\beta}\right)=d_{\mathbb{R}^{n}}\left(-\omega_{i}^{\alpha} d x^{i}\right)=\mathscr{D}_{i, j}^{\alpha} d x^{i} d x^{j}\left(\omega_{k}^{\beta} d x^{k} \otimes \eta_{\beta}\right) .
$$

Again foreshadowing, $\mathscr{D}_{i, j}^{\alpha} d x^{i} d x^{j}$ will be denoted by $\partial \rho^{\alpha}$, and thus

$$
\partial \rho^{\alpha}\left(\omega_{k}^{\beta} d x^{k} \otimes \eta_{\beta}\right)=-d_{\mathbb{R}^{n}}\left(\omega_{i}^{\alpha} d x^{i}\right) .
$$

Another notational simplification will be used from now on. The above operators, when evaluated, will be written as: $\eta^{\alpha}\left(f^{\beta} \eta_{\beta}\right)=f^{\alpha}, \partial \eta^{\alpha}\left(f^{\beta} \eta_{\beta}\right)=d_{\mathbb{R}^{n}} f^{\alpha}$, $\rho^{\alpha}\left(\omega^{\beta} \eta_{\beta}\right)=-\omega^{\alpha}$, and $\partial \rho^{\alpha}\left(\omega^{\beta} \eta_{\beta}\right)=-d_{\mathbb{R}^{n}} \omega^{\alpha}$. Note that $f^{\alpha}$ is a function, while $d_{\mathbb{R}^{n}} f^{\alpha}$ and $\omega^{\alpha}$ are one-forms, and $d_{\mathbb{R}^{n}} \omega^{\alpha}$ is a two-form. Finally, it will be convenient to have symbols to refer to the various vector spaces spanned by these four types of operators. The vector spaces will be denoted by $\eta, \partial \eta, \rho$, and $\partial \rho$. Thus if superscripts occur, the operator is being referred to; whereas if no superscript occurs, the vector space is being referred to. Notice the degrees of the various vector spaces: $\operatorname{deg}(\eta)=1, \operatorname{deg}(\partial \eta)=2, \operatorname{deg}(\rho)=1$, and

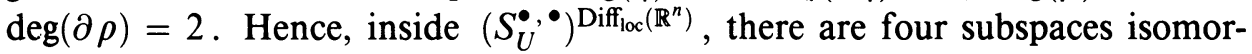
phic to $\mathfrak{g}^{*}$, two of degree one and two of degree two. This observation will play an important role in what follows.

\section{The Algebra of OPERATORS INVARIANT UNDER $\operatorname{Diff}_{\text {loc }}\left(\mathbb{R}^{n}\right)$}

Consider the graded vector space $V=\eta \oplus \partial \eta \oplus \rho \oplus \partial \rho$, where $V^{1}=\eta \oplus \rho$, $V^{2}=\partial \eta \oplus \partial \rho$, and $V^{i}=0$, if $i \neq 1,2$ and its graded commutative algebra, $\Lambda(\eta \oplus \partial \eta \oplus \rho \oplus \partial \rho)$. Since both $\eta$ and $\rho$ are of degree one, while $\partial \eta$ and $\partial \rho$ are of degree two, the graded product of their elements is completely determined and there are the isomorphisms,

$$
\begin{aligned}
\Lambda(\eta \oplus \partial \eta \oplus \rho \oplus \partial \rho) & \simeq E(\eta \oplus \rho) \otimes S(\partial \eta \oplus \partial \rho) \\
& \simeq E(\eta) \otimes S(\partial \eta) \otimes E(\rho) \otimes S(\partial \rho) .
\end{aligned}
$$

Furthermore, $\Lambda(\eta \oplus \partial \eta \oplus \rho \oplus \partial \rho)$ is well defined without reference to the base manifold, since, as was pointed out in $\S 6$, the vector spaces $\eta, \partial \eta, \rho$, and $\partial \rho$ are well defined independently of $M$. This graded algebra is universal in the sense that, given a base manifold $M$ of dimension $n$, there is a graded algebra homomorphism $\Pi: \Lambda(\eta \oplus \partial \eta \oplus \rho \oplus \partial \rho) \rightarrow S_{U}^{\bullet, \bullet}$, where $U$ is an open subset of $M$. Because the local diffeomorphisms act as algebra homomorphisms, i.e.

$$
\Phi^{*}\left(\eta^{\alpha}+\eta^{\beta}\right)=\Phi^{*}\left(\eta^{\alpha}\right)+\Phi^{*}\left(\eta^{\beta}\right) \text { and } \Phi^{*}\left(\eta^{\alpha} \wedge \eta^{\beta}\right)=\Phi^{*}\left(\eta^{\alpha}\right) \wedge \Phi^{*}\left(\eta^{\beta}\right)
$$

(similarly for all other sums and products), the image of $\Lambda(\eta \oplus \partial \eta \oplus \rho \oplus \partial \rho)$ is contained in $\left(S_{U}^{\bullet, \bullet}\right)^{\text {Diffloc }}\left(\mathbb{R}^{n}\right)$. Recall that $\eta^{\alpha}$ has form degree zero, $\partial \eta^{\alpha}$ has form degree one, $\rho^{\alpha}$ has form degree one, and $\partial \rho^{\alpha}$ has form degree two. This implies that the image of an element in

$$
E^{m}(\eta) \otimes S^{r}(\partial \eta) \otimes E^{s}(\rho) \otimes S^{t}(\partial \rho)
$$


(a graded product of $m \eta^{\alpha}$ 's, $r \partial \eta^{\alpha}$ 's, $s \rho^{\alpha}$ 's and $t \partial \rho^{\alpha}$ 's), will be zero, if $r+s+2 t>n$, since its form degree will be larger than the dimension of the manifold. Furthermore, beyond the relations arising from the exterior algebras, this is the only new relation between the products of operators in these four vector spaces, once the dimension of the base space $M$ has been specified. This follows because, as long as the form degree of a product is less than the dimension of the base space $M$, a product vanishing implies its vanishing for all $f^{\prime} s \in \Omega^{0}(U, \mathfrak{g})$ and $\omega^{\prime} s \in \Omega^{1}(U, \mathfrak{g})$. But clearly this is impossible for a nonzero operator, since the value of a product is the wedge product of functions $f^{\alpha}$, one-forms $d_{\mathbb{R}^{n}} f^{\alpha}$ and $\omega^{\alpha}$, and two-forms $d_{\mathbb{R}^{n}} \omega^{\alpha}$, where the $f^{\alpha}$ 's and $\omega^{\alpha}$ 's are arbitrary.

Another way to express this is that the kernel of the graded algebra homomorphism $\Pi$ is the ideal $I$, generated by the subspaces $S^{r}(\partial \eta) \otimes E^{s}(\rho) \otimes S^{t}(\partial \rho)$, where $r+s+2 t>n$. This ideal $I$ will be denoted by

$$
\left(\bigoplus_{r+s+2 t>n} S^{r}(\partial \eta) \otimes E^{s}(\rho) \otimes S^{t}(\partial \rho)\right),
$$

and the quotient will be denoted by $\Lambda(\eta \oplus \partial \eta \oplus \rho \oplus \partial \rho)_{q \leq n}$. Recall that $q$ denotes the form index of $S_{U}^{p, q}$. Furthermore, the inclusion of the quotient into $S_{U}^{\bullet, \bullet}$ is injective. The importance of the graded algebra $\Lambda(\eta \oplus \partial \eta \oplus \rho \oplus \partial \rho)_{q \leq n}$ is contained in the following theorem.

Theorem. The graded algebra $\Lambda(\eta \oplus \partial \eta \oplus \rho \oplus \partial \rho)_{q \leq n}$ is isomorphic to the graded algebra $\left(S_{U}^{\bullet, \bullet}\right)^{A(n)}$. Therefore there is the sequence of relations

$$
\begin{gathered}
\left(S_{U}^{\bullet, \bullet}\right)^{\text {Diff }_{\text {loc }}\left(\mathbb{R}^{n}\right)} \subset\left(S_{U}^{\bullet, \bullet}\right)^{A(n)} \simeq \Lambda(\eta \oplus \partial \eta \oplus \rho \oplus \partial \rho)_{q \leq n} \\
\simeq \Pi(\Lambda(\eta \oplus \partial \eta \oplus \rho \oplus \partial \rho)) \subset\left(S_{U}^{\bullet, \bullet}\right)^{\operatorname{Diff}_{\text {loc }}\left(\mathbb{R}^{n}\right)},
\end{gathered}
$$

which imply that $\Lambda(\eta \oplus \partial \eta \oplus \rho \oplus \partial \rho)_{q \leq n}$ is isomorphic to $\left(S_{U}^{\bullet, \bullet}\right)^{\operatorname{Diffloc}_{\text {loc }}\left(\mathbb{R}^{n}\right)}$.

Proof. Recall that a typical basis element of the invariants of $\S 5$ is of the form

$$
D_{\left(i_{1}, \ldots, i_{s}, 0, \ldots, 0\right)}^{\left(\alpha_{1}, \ldots, \alpha_{p}\right)} \mathscr{D}_{\left(i_{s+1}, \ldots, i_{t}, 0, \ldots, 0\right),\left(i_{t+1}, \ldots, i_{q}\right)}^{\left(\beta_{1}, \ldots, \mathbf{x}_{m}\right)} d \mathbf{x}^{\left(i_{1}, \ldots, i_{q}\right)} .
$$

By using the graded product of $S_{U}^{\bullet}, \bullet$, it follows that the basis element

$$
D_{\left(i_{1}, \ldots, i_{s}, 0, \ldots, 0\right)}^{\left(\alpha_{1}, \ldots, \alpha_{p}\right)} \mathscr{D}_{\left(i_{s+1}, \ldots, i_{t}, 0, \ldots, 0\right),\left(i_{t+1}, \ldots, i_{q}\right)}^{\left(\beta_{1}, \ldots, \beta_{m}\right)} d \mathbf{x}^{\left(i_{1}, \ldots, i_{q}\right)} \in S^{p, q}
$$

is the product

$$
=\left(D_{\left(i_{1}, \ldots, i_{s}, 0, \ldots, 0\right)}^{\left(\alpha_{1}, \ldots, \alpha_{p}\right)} d \mathbf{x}^{\left(i_{1}, \ldots, i_{s}\right)}\right) \wedge\left(\mathscr{D}_{\left(i_{s+1}, \ldots, i_{t}, 0, \ldots, 0\right),\left(i_{t+1}, \ldots, i_{q}\right)}^{\left(\beta_{1}, \ldots, \beta_{m}\right)} d \mathbf{x}^{\left(i_{s+1}, \ldots, i_{q}\right)}\right) .
$$

Note that the first term, $D_{\left(i_{1}, \ldots, i_{s}, 0, \ldots, 0\right)}^{\left(\alpha_{1}, \ldots, \alpha_{p}\right)} d \mathbf{x}^{\left(i_{1}, \ldots, i_{s}\right)} \in S_{U}^{p, s}$, is homogeneous of degree zero, i.e. $|B|=0$, and the second term,

$$
\mathscr{D}_{\left(i_{s+1}, \ldots, i_{t}, 0, \ldots, 0\right),\left(i_{t+1}, \ldots, i_{q}\right)}^{\left(\beta_{1}, \ldots, \beta_{m}\right)} d \mathbf{x}^{\left(i_{s+1}, \ldots, i_{q}\right)} \in S_{U}^{0, q-s},
$$

has vertical degree zero. Again, using the $\wedge$ of $S_{U}^{\bullet, \bullet}$, the first operator

$$
D_{\left(i_{1}, \ldots, i_{s}, 0, \ldots, 0\right)}^{\left(\alpha_{1}, \ldots, \alpha_{p}\right)} d \mathbf{x}^{\left(i_{1}, \ldots, i_{s}\right)}
$$

decomposes as $(-1)^{s(p-s)}\left(D_{\left(i_{1}, \ldots, i_{s}\right)}^{\left(\alpha_{1}, \ldots, \alpha_{s}\right)} d \mathbf{x}^{\left(i_{1}, \ldots, i_{s}\right)}\right) \wedge\left(D_{(0, \ldots, 0)}^{\left(\alpha_{s+1}, \ldots, \alpha_{p}\right)}\right)$, but the first factor decomposes as

$$
\pm\left(D_{i_{1}}^{\alpha_{1}} d x^{i_{1}}\right) \wedge \cdots \wedge\left(D_{i_{s}}^{\alpha_{s}} d x^{i_{s}}\right)= \pm \partial \eta^{\alpha_{1}} \wedge \cdots \wedge \partial \eta^{\alpha_{s}}
$$


while the second factor is

$$
\left(D_{0}^{\alpha_{s+1}}\right) \wedge \cdots \wedge\left(D_{0}^{\alpha_{p}}\right)=\eta^{\alpha_{s+1}} \wedge \cdots \wedge \eta^{\alpha_{p}} .
$$

On the other hand, the second operator $\mathscr{D}_{\left(i_{s+1}, \ldots, i_{t}, 0, \ldots, 0\right),\left(i_{t+1}, \ldots, i_{q}\right)}^{\left(\beta_{1}, \ldots, \beta_{m}\right)} d \mathbf{x}^{\left(i_{s+1}, \ldots, i_{q}\right)}$ decomposes as

$$
\left(\mathscr{D}_{\left(i_{s+1}, \ldots, i_{t}\right),\left(i_{t+1}, \ldots, i_{2 t}\right)}^{\left(\beta_{1}, \ldots, \beta_{t^{\prime}}\right.} d \mathbf{x}^{\left(i_{s+1}, \ldots, i_{2 t}\right)}\right) \wedge\left(D_{(0, \ldots, 0),\left(i_{2 t+1}, \ldots, i_{q}\right)}^{\left(\beta_{t+1}, \ldots, \beta_{m}\right)} d \mathbf{x}^{\left(i_{2 t+1}, \ldots, i_{q}\right)}\right),
$$

but the operator $\mathscr{D}_{\left(i_{s+1}, \ldots, i_{t}\right),\left(i_{t+1}, \ldots, i_{2 t}\right)}^{\left(\beta_{1}, \ldots, \beta_{t}\right)} d \mathbf{x}^{\left(i_{s+1}, \ldots, i_{2 t}\right)}$ decomposes as

$$
\pm\left(\mathscr{D}_{i_{s+1}, i_{t+1}}^{\beta_{1}} d x^{i_{s+1}} d x^{i_{t+1}}\right) \wedge \cdots \wedge\left(\mathscr{D}_{i_{t}, i_{2 t}}^{\beta_{t}} d x^{i_{t}} d x^{i_{2 t}}\right)= \pm \partial \rho^{\beta_{1}} \wedge \cdots \wedge \partial \rho^{\beta_{t}},
$$

while the operator $\mathscr{D}_{(0, \ldots, 0),\left(i_{2 t+1}, \ldots, i_{q}\right)}^{\left(\beta_{t+1}, \ldots, \beta_{m}\right)} d \mathbf{x}^{\left(i_{2 t+1}, \ldots, i_{q}\right)}$ decomposes as

$$
\pm\left(\mathscr{D}_{0, i_{2 t+1}}^{\beta_{t+1}} d x^{i_{2 t+1}}\right) \wedge \cdots \wedge\left(\mathscr{D}_{0, i_{q}}^{\beta_{m}} d x^{i_{q}}\right)= \pm \rho^{\beta_{t+1}} \wedge \cdots \wedge \rho^{\beta_{m}}
$$

Thus it follows that the basis element

$$
\begin{aligned}
D_{\left(i_{1}, \ldots, i_{s}, 0, \ldots, 0\right)}^{\left(\alpha_{1}, \ldots, \alpha_{p}\right)} & \mathscr{D}_{\left(i_{s+1}, \ldots, i_{t}, 0, \ldots, 0\right),\left(i_{t+1}, \ldots, i_{q}\right)}^{\left(\beta_{1}, \ldots \mathbf{\beta}\right)} d \mathbf{x}^{\left(i_{1}, \ldots, i_{q}\right)} \\
= & \left(\partial \eta^{\alpha_{1}} \wedge \cdots \wedge \partial \eta^{\alpha_{p}}\right) \wedge\left(\eta^{\alpha_{s+1}} \wedge \cdots \wedge \eta^{\alpha_{s}}\right) \\
& \wedge\left(\partial \rho^{\beta_{1}} \wedge \cdots \wedge \partial \rho^{\beta_{t}}\right) \wedge\left(\rho^{\beta_{t+1}} \wedge \cdots \wedge \rho^{\beta_{m}}\right)
\end{aligned}
$$

with at most a sign change. Thus every operator, satisfying the requirements of $\S 5$, is in the image of $\Lambda(\eta \oplus \partial \eta \oplus \rho \oplus \partial \rho)_{q \leq n}$, and therefore is invariant under all of Diff loc $\left(\mathbb{R}^{n}\right)$. This proves the theorem and finishes the search for and description of $\left(S_{U}^{\bullet, \bullet}\right)^{\text {Diffloc }}\left(\mathbb{R}^{n}\right)$.

\section{The Differentials $\partial, \delta, d$ of $\Lambda(\eta \oplus \partial \eta \oplus \rho \oplus \partial \rho)$}

In this section the differentials $\delta$ and $\partial$ of the differential graded algebra $S_{M}^{\bullet}{ }^{\bullet}$ will be translated in Theorems $8.1,8.2$, and 8.3 to the graded algebra $\Lambda(\eta \oplus \partial \eta \oplus \rho \oplus \partial \rho)$, by determining the action of $\partial$ and $\delta$ on the generators $\eta^{\alpha}, \partial \eta^{\alpha}, \rho^{\alpha}$ and $\partial \rho^{\alpha}$. It will be shown that with respect to the total differential $d=\partial+(-1)^{q} \delta$, the graded algebra $\Lambda(\eta \oplus \partial \eta \oplus \rho \oplus \partial \rho)$ is isomorphic to a twisted tensor product of $W(\mathfrak{g})$ and $K(\mathfrak{g})$.

Theorem 8.1. The graded algebra $\Lambda(\eta \oplus \partial \eta \oplus \rho \oplus \partial \rho)$ with the differential $\partial$ is isomorphic to the tensor product of two copies of $K(\mathfrak{g})$. Hence it is acyclic with respect to $\partial$.

Proof. Via the map of the generators of $\Lambda(\eta \oplus \partial \eta \oplus \rho \oplus \partial \rho)$ into $S_{U}^{\bullet, \bullet}$, the differential $\partial$ of $S_{U}^{\bullet, \bullet}$ induces a differential $\partial$ on $\Lambda(\eta \oplus \partial \eta \oplus \rho \oplus \partial \rho)$. Let $\partial$ also denote the induced differential. On the one hand, with malice aforethought of notation, it follows that $\partial\left(\eta^{\alpha}\right)=\partial \eta^{\alpha}$ and $\partial\left(\rho^{\alpha}\right)=\partial \rho^{\alpha}$. On the other hand, the induced differential $\partial$ on $\partial \eta^{\alpha}$ and $\partial \rho^{\alpha}$ is defined by $\left(\partial \partial \eta^{\alpha}\right)(f)=$ $d_{\mathbb{R}^{n}}^{2}\left(f^{\alpha}\right)=0$ and $\left(\partial \partial \rho^{\alpha}\right)(\omega)=d_{\mathbb{R}^{n}}^{2}\left(\omega^{\alpha}\right)=0$, for $f \in \Omega^{0}(U, \mathfrak{g})$ and $\omega \in$ $\Omega^{1}(U, \mathfrak{g})$. Thus $\partial$ is defined on the generators of $\Lambda(\eta \oplus \partial \eta \oplus \rho \oplus \partial \rho)$ by $\partial\left(\eta^{\alpha}\right)=\partial \eta^{\alpha}, \partial\left(\partial \eta^{\alpha}\right)=0, \partial\left(\rho^{\alpha}\right)=\partial \rho^{\alpha}, \partial\left(\partial \rho^{\alpha}\right)=0$. This implies that $\Lambda(\eta \oplus \partial \eta \oplus \rho \oplus \partial \rho) \simeq \Lambda(\eta \oplus \partial \eta) \otimes \Lambda(\rho \oplus \partial \rho)$ with the differential $\partial$ is the tensor product of two copies of $K(\mathfrak{g})$, hence it is acyclic. 
Theorem 8.2. The graded subalgebra $\Lambda(\eta \oplus \partial \eta)$ with the differential $\partial+(-1)^{q} \delta$ is isomorphic to $W(\mathfrak{g})$.

Proof. Since both $\eta^{\alpha}$ and $\partial \eta^{\alpha}$ have homogeneous degree zero and vertical degree one, the induced differential $\delta$ is

$$
\begin{aligned}
\left(\delta \eta^{\alpha}\right)\left(f^{\beta} \eta_{\beta}, g^{\gamma} \eta_{\gamma}\right) & =-\eta^{\alpha}\left(\left[f^{\beta} \eta_{\beta}, g^{\gamma} \eta_{\gamma}\right]\right)=-C_{\beta \gamma}^{\alpha} f^{\beta} g^{\gamma} \\
& =-\frac{1}{2} C_{\beta \gamma}^{\alpha} \eta^{\gamma} \wedge \eta^{\beta}\left(f^{\sigma} \eta_{\sigma}, g^{\tau} \eta_{\tau}\right),
\end{aligned}
$$

and

$$
\begin{aligned}
\left(\delta \partial \eta^{\alpha}\right)\left(f^{\beta} \eta_{\beta}, g^{\gamma} \eta_{\gamma}\right) & =-\partial \eta^{\alpha}\left(\left[f^{\beta} \eta_{\beta}, g^{\gamma} \eta_{\gamma}\right]\right)=-d_{\mathbb{R}^{n}}\left(C_{\beta \gamma}^{\alpha} f^{\beta} g^{\gamma}\right) \\
& =C_{\beta \gamma}^{\alpha} \eta^{\gamma} \wedge \partial \eta^{\beta}\left(f^{\sigma} \eta_{\sigma}, g^{\tau} \eta_{\tau}\right) .
\end{aligned}
$$

This is precisely the extension of the Chevalley-Eilenberg differential $\delta$ to $W(\mathfrak{g})$, when $\sigma \mathfrak{g}^{*}$ is identified with $\partial \eta$. Combining this with the result of Theorem 8.1, that $\partial$ is the same as the Koszul differential of $K(\mathfrak{g})$, it follows that $\Lambda(\eta \oplus \partial \eta)$ with the differentials $d=\partial+(-1)^{q} \delta$ is isomorphic to $W(\mathfrak{g})$. Note that this isomorphism is not just at the cohomology level, but at the differential graded algebra level as well.

Theorem 8.3. The differential $\delta$ when restricted to $\Lambda(\rho \oplus \partial \rho)$ decomposes into two differentials. The first differential is induced by the "co-adjoint" action, denoted by $\vartheta$, of $\Omega^{0}(U, \mathfrak{g})$ on $\rho$ and $\partial \rho$, where $\left(\vartheta(f) \rho^{\alpha}\right)(\omega)=-\rho^{\alpha}([\omega, f])=$ $-\rho^{\alpha}\left(C_{\beta \gamma}^{\sigma} \omega^{\beta} f^{\gamma} \eta_{\sigma}\right)$, and similarly for $\partial \rho$. The second differential $\tau$ sends $\rho^{\alpha}$ to $\partial \eta^{\alpha}$ and $\partial \rho^{\alpha}$ to 0 .

Proof. The fact that for trivial bundles $U \times G, A_{0}$ may be chosen so that $d_{A_{0}} f=d_{\mathbb{R}^{n}} f$ [8] will be used in the following calculation. This is important because both $\rho^{\alpha}$ and $\partial \rho^{\alpha}$ have vertical degree zero, and thus the differential $\delta$ reduces to the action of $\theta(f)$ on $\rho^{\alpha}$ and $\partial \rho^{\alpha}$ for $f \in \Omega^{0}(U, \mathfrak{g})$. Another important property of both $\rho^{\alpha}$ and $\partial \rho^{\alpha}$ is that, since they both have homogeneous degree one, they are linear in $\omega$.

From the definition of $\delta$ on $S_{U}^{\bullet} \bullet$, it follows that the induced $\delta$ on $\Lambda(\rho \oplus \partial \rho)$ is defined $\rho^{\alpha}$ and $\delta \rho^{\alpha}$ by

$$
\begin{aligned}
\left(\delta \rho^{\alpha}\right)(f, \omega) & =-\left.\frac{d}{d t} \rho^{\alpha}\left(\omega+t\left(d_{\mathbb{R}^{n}} f+[\omega, f]\right)\right)\right|_{t=0} \\
& =d_{\mathbb{R}^{n}} f^{\alpha}+C_{\beta \gamma}^{\alpha} \omega^{\beta} f^{\gamma}=\partial \eta^{\alpha}(f)-\rho^{\alpha}([\omega, f]),
\end{aligned}
$$

and

$$
\begin{aligned}
\left(\delta \partial \rho^{\alpha}\right)(f, \omega) & =-\left.\frac{d}{d t} \partial \rho^{\alpha}\left(\omega+t\left(d_{\mathbb{R}^{n}} f+[\omega, f]\right)\right)\right|_{t=0} \\
& =d_{\mathbb{R}^{n}}^{2} f^{\alpha}+d_{\mathbb{R}^{n}}\left(C_{\beta \gamma}^{\alpha} \omega^{\beta} f^{\gamma}\right)=-\partial \rho^{\alpha}([\omega, f])
\end{aligned}
$$

for $f \in \Omega^{0}(U, \mathfrak{g})$ and $\omega \in \Omega^{1}(U, \mathfrak{g})$. Thus, on the generators $\rho^{\alpha}$ and $\partial \rho^{\alpha}$, the differential $\delta$ decomposes into the differential $\tau$, where $\tau\left(\rho^{\alpha}\right)=\partial \eta^{\alpha}$, while $\tau\left(\partial \rho^{\alpha}\right)=0$, and the co-adjoint action $\vartheta$ of $f \in \Omega^{0}(U, \mathfrak{g})$ on $\rho^{\alpha}$ and $\partial \rho^{\alpha}$.

Theorem 8.4. The differential graded algebra $\Lambda(\eta \oplus \partial \eta \oplus \rho \oplus \partial \rho)$ with the differential $d=\partial+(-1)^{q} \delta$ is isomorphic to a twisted tensor product of $W(\mathfrak{g})$ 
and $K(\mathfrak{g})$, denoted by $W(\mathfrak{g}) \otimes_{\tau} K(\mathfrak{g})$, where $\otimes_{\tau}$ means twisted tensor product. Furthermore, it is acyclic with respect to $d$.

Proof. The total differential $d$ has the following values on the generators:

$$
\begin{gathered}
d\left(\eta^{\alpha}\right)=\partial \eta^{\alpha}-\frac{1}{2} C_{\beta \gamma}^{\alpha} \eta^{\gamma} \wedge \eta^{\beta}, \quad d\left(\partial \eta^{\alpha}\right)=-C_{\beta \gamma}^{\alpha} \eta^{\gamma} \wedge \partial \eta^{\beta}, \\
d\left(\rho^{\alpha}\right)=\partial \rho^{\alpha}-\partial \eta^{\alpha}-C_{\beta \gamma}^{\alpha} \eta^{\gamma} \wedge \rho^{\beta}
\end{gathered}
$$

and

$$
d\left(\partial \rho^{\alpha}\right)=C_{\beta \gamma}^{\alpha}\left(\partial \eta^{\gamma} \wedge \rho^{\beta}-\eta^{\gamma} \wedge \partial \rho^{\beta}\right) .
$$

On the one hand, the inclusion of $W(\mathfrak{g})$ in $\Lambda(\eta \oplus \partial \eta \oplus \rho \oplus \partial \rho)$, given by

$$
W(\mathfrak{g}) \simeq \Lambda(\eta \oplus \partial \eta) \hookrightarrow \Lambda(\eta \oplus \partial \eta \oplus \rho \oplus \partial \rho),
$$

is a differential graded algebra homomorphism. On the other hand, there is the projection

$$
\Lambda(\eta \oplus \partial \eta \oplus \rho \oplus \partial \rho) / \Lambda^{+}(\eta \oplus \partial \eta) \rightarrow \Lambda(\rho \oplus \partial \rho)
$$

where the latter graded algebra, with the differential $\partial$, was seen to be isomorphic to $K(\mathfrak{g})$. Note that the total differential $d$ becomes $\partial$ under the above projection and that the differential $\tau$ decreases the $\rho$ degree by one, i.e.

$$
\tau: \Lambda^{n}(\rho \oplus \partial \rho) \rightarrow \Lambda(\eta \oplus \partial \eta) \otimes \Lambda^{n-1}(\rho \oplus \partial \rho) .
$$

Thus there is the following diagram of differential graded algebras:

$$
\Lambda(\eta \oplus \partial \eta) \hookrightarrow \Lambda(\eta \oplus \partial \eta \oplus \rho \oplus \partial \rho) \rightarrow \Lambda(\rho \oplus \partial \rho)
$$

But by the definition of a twisted tensor product of differential graded algebras $[11,16]$, this implies that

$$
\Lambda(\eta \oplus \partial \eta \oplus \rho \oplus \partial \rho) \simeq W(\mathfrak{g}) \otimes_{\tau} K(\mathfrak{g}),
$$

where the base algebra is $W(\mathfrak{g})$, the total algebra is $\Lambda(\eta \oplus \partial \eta \oplus \rho \oplus \partial \rho)$, the fiber is $K(\mathfrak{g})$, and the twisting differential is $\tau$.

Finally, as was noted before in Theorem $8.1, \Lambda(\eta \oplus \partial \eta \oplus \rho \oplus \partial \rho)$ is acyclic with respect to the differential $\partial$ but this implies that in the spectral sequence, where $d_{0}=\partial$, the $E_{1}$ term is zero. Thus $E_{\infty}$ is also 0 and $\Lambda(\eta \oplus \partial \eta \oplus \rho \oplus \partial \rho)$ is acyclic relative to the total differential $d$.

Theorem 8.5. The differential graded algebra $\Lambda(\eta \oplus \partial \eta \oplus \rho \oplus \partial \rho)$ is a $\mathfrak{g}$-differential graded algebra with respect to the differential $d$.

Proof. Notice that $\mathfrak{g}$ is contained in $\Omega^{0}(U, \mathfrak{g})$, where $\mathfrak{g}$ is identified with those sections of $\Omega^{0}(U, \mathfrak{g})$ which are constant. Let $x=x^{\alpha} \eta_{\alpha} \in \mathfrak{g}$ and identify the $x^{\alpha}$ as the constant functions on $U$. Let $i$ be defined on the generators as

$$
i(x) \eta^{\alpha}=x^{\alpha}, \quad i(x) \partial \eta^{\alpha}=d x^{\alpha}=0, \quad i(x) \rho^{\alpha}=0, \quad \text { and } \quad i(x) \partial \rho^{\alpha}=0,
$$

while define $\theta$ on the generators as

$$
\theta(x) \eta^{\alpha}=-C_{\beta \gamma}^{\alpha} x^{\gamma} \eta^{\beta}, \quad \theta(x) \partial \eta^{\alpha}=-C_{\beta \gamma}^{\alpha} x^{\gamma} \partial \eta^{\beta}, \quad \theta(x) \rho^{\alpha}=-C_{\beta \gamma}^{\alpha} x^{\gamma} \rho^{\beta},
$$

and

$$
\theta(x) \partial \rho^{\alpha}=-C_{\beta \gamma}^{\alpha} x^{\gamma} \partial \rho^{\beta}
$$

Note that $\theta$ is just the restriction of $\vartheta$ to the constant functions. Since $x^{\gamma}$ is a constant, it follows that $\theta(x)$ is a well-defined endomorphism for each of 
the vector spaces $\eta, \partial \eta, \rho$, and $\partial \rho$. As usual, $i$ and $\theta$ are extended as derivations of all of $\Lambda(\eta \oplus \partial \eta \oplus \rho \oplus \partial \rho)$. It is now an easy exercise in the definitions of $i, \theta$, and $d$ to check that $i([x, y])=\theta(x) i(y)-i(x) \theta(y)$ and $\theta(x)=d i(x)+i(x) d$. This proves the theorem.

\section{THE COHOMOLOGY OF $\Lambda(\eta \oplus \partial \eta \oplus \rho \oplus \partial \rho)_{q \leq n}$}

Theorem 8.5 proved that the graded algebra of invariants of the pseudo-group of local diffeomorphisms, with the differential $d$, is a $\mathfrak{g}$-differential graded algebra. Consider the algebraic connection $A: \mathfrak{g}^{*} \rightarrow \Lambda(\eta \oplus \partial \eta \oplus \rho \oplus \partial \rho)$, given by $A\left(\eta^{\alpha}\right)=\eta^{\alpha}+\rho^{\alpha}$, where $\eta^{\alpha}$ is playing a double role as a basis element of $\mathfrak{g}^{*}$ and as a generator of $\Lambda(\eta \oplus \partial \eta \oplus \rho \oplus \partial \rho)$. This is a connection because of the identities: $i(x) A\left(\eta^{\alpha}\right)=i(x)\left(\eta^{\alpha}+\rho^{\alpha}\right)=\eta^{\alpha}(x)+0=x^{\alpha}$, and

$$
\begin{aligned}
\theta(x) A\left(\eta^{\alpha}\right) & =\theta(x)\left(\eta^{\alpha}+\rho^{\alpha}\right)=-C_{\beta \gamma}^{\alpha} x^{\gamma} \eta^{\beta}+-C_{\beta \gamma}^{\alpha} x^{\gamma} \rho^{\beta} \\
& =-C_{\beta \gamma}^{\alpha} x^{\gamma}\left(\eta^{\beta}+\rho^{\beta}\right)=A\left(-C_{\beta \gamma}^{\alpha} x^{\gamma} \eta^{\beta}\right)=A\left(\theta(x) \eta^{\alpha}\right),
\end{aligned}
$$

where, as before, $\mathfrak{g}$ has been identified with the constant sections of $\Omega^{0}(U, \mathfrak{g})$.

Theorem 9 (The main theorem). For any compact, connected Lie group $G$ with Lie algebra $\mathfrak{g}$, there is a chain map

$$
\tilde{\varpi}:\left(S_{U}^{\bullet, \bullet}\right)^{\operatorname{Diffloc}_{\text {loc }}\left(\mathbb{R}^{n}\right)} \rightarrow W(\mathfrak{g})_{\left[\frac{n}{2}\right]}
$$

which induces an isomorphism in cohomology where $W(\mathfrak{g})_{\left[\frac{n}{2}\right]}$ is the Weil algebra truncated at the greatest integer less than or equal to half the dimension of the manifold. Thus for any compact, connected Lie group $G$, with Lie algebra $\mathfrak{g}$, there is an isomorphism

$$
H_{d}^{\bullet}\left(\left(S_{U}^{\bullet, \bullet}\right)^{\operatorname{Diff}_{\text {loc }}\left(\mathbb{R}^{n}\right)}\right) \simeq H_{d}^{\bullet}\left(W(\mathfrak{g})_{\left[\frac{n}{2}\right]}\right) .
$$

Furthermore, $H_{d}^{q}\left(\left(S_{U}^{\bullet, \bullet}\right)^{\operatorname{Diff}_{\mathrm{loc}}\left(\mathbb{R}^{n}\right)}\right)$ is zero if $q<n$ and $n+r<q$ where $n$ is the dimension of $U, r$ is the dimension of $\mathfrak{g}$ and if $n \leq q \leq n+r$ then the dimension of $H_{d}^{q}\left(\left(S_{U}^{\bullet, \bullet}\right)^{\operatorname{Diff}_{\mathrm{loc}}\left(\mathbb{R}^{n}\right)}\right)$ is finite.

Proof. Start with the above connection $A$, namely $A\left(\eta^{\alpha}\right)=\eta^{\alpha}+\rho^{\alpha}$. The following calculation determines the value of $(A, F)$ on $\sigma \eta$,

$$
\begin{aligned}
(A, F)\left(\sigma \eta^{\alpha}\right)= & d A\left(\eta^{\alpha}\right)-A\left(\delta \eta^{\alpha}\right)=d\left(\eta^{\alpha}+\rho^{\alpha}\right)-A\left(-\frac{1}{2} C_{\beta \gamma}^{\alpha} \eta^{\gamma} \wedge \eta^{\beta}\right) \\
= & \left(\partial \eta^{\alpha}-\frac{1}{2} C_{\beta \gamma}^{\alpha} \eta^{\gamma} \wedge \eta^{\beta}\right)+\left(\partial \rho^{\alpha}-\partial \eta^{\alpha}-C_{\beta \gamma}^{\alpha} \eta^{\gamma} \wedge \rho^{\beta}\right) \\
& +\frac{1}{2} C_{\beta \gamma}^{\alpha}\left(\eta^{\gamma}+\rho^{\gamma}\right) \wedge\left(\eta^{\beta}+\rho^{\beta}\right)=\partial \rho^{\alpha}-\frac{1}{2} C_{\beta \gamma}^{\alpha} \eta^{\gamma} \wedge \eta^{\beta}-C_{\beta \gamma}^{\alpha} \eta^{\gamma} \wedge \rho^{\beta} \\
& +\frac{1}{2} C_{\beta \gamma}^{\alpha}\left(\left(\eta^{\gamma} \wedge \eta^{\beta}\right)+\left(\eta^{\gamma} \wedge \rho^{\beta}\right)+\left(\rho^{\gamma} \wedge \eta^{\beta}\right)+\left(\rho^{\gamma} \wedge \rho^{\beta}\right)\right) \\
= & \partial \rho^{\alpha}+\frac{1}{2} C_{\beta \gamma}^{\alpha} \rho^{\gamma} \wedge \rho^{\beta} .
\end{aligned}
$$

Thus the image of $W(\mathfrak{g})$ is generated by the elements $\eta^{\alpha}+\rho^{\alpha}$ and $\partial \rho^{\alpha}+$ $\frac{1}{2} C_{\beta \gamma}^{\alpha} \rho^{\gamma} \wedge \rho^{\beta}$, where the former has total degree one and the latter has total degree two. This implies that the subalgebra of $\Lambda(\eta \oplus \partial \eta \oplus \rho \oplus \partial \rho)$ generated by these elements is isomorphic to $\Lambda\left(\mathfrak{g}^{*} \oplus \sigma \mathfrak{g}^{*}\right)$. Finally, since $d(A, F)=(A, F) d$, by the fact that $(A, F)$ is always a chain map, it follows that:

$$
\begin{aligned}
d\left(\eta^{\alpha}+\rho^{\alpha}\right) & =d(A, F)\left(\eta^{\alpha}\right)=(A, F)\left(d \eta^{\alpha}\right)=(A, F)\left(\sigma \eta^{\alpha}-\frac{1}{2} C_{\beta \gamma}^{\alpha} \eta^{\gamma} \wedge \eta^{\beta}\right) \\
& =\left(\partial \rho^{\alpha}+\frac{1}{2} C_{\beta \gamma}^{\alpha} \rho^{\gamma} \wedge \rho^{\beta}\right)-\frac{1}{2} C_{\beta \gamma}^{\alpha}\left(\eta^{\gamma}+\rho^{\gamma}\right) \wedge\left(\eta^{\beta}+\rho^{\beta}\right),
\end{aligned}
$$


and

$$
\begin{aligned}
d\left(\partial \rho^{\alpha}+\frac{1}{2} C_{\beta \gamma}^{\alpha} \rho^{\gamma} \wedge \rho^{\beta}\right) & =d(A, F)\left(\sigma \eta^{\alpha}\right)=(A, F)\left(d \sigma \eta^{\alpha}\right) \\
& =(A, F)\left(-C_{\beta \gamma}^{\alpha} \eta^{\gamma} \wedge \sigma \eta^{\beta}\right) \\
& =-C_{\beta \gamma}^{\alpha}\left(\eta^{\gamma}+\rho^{\gamma}\right) \wedge\left(\partial \rho^{\beta}+\frac{1}{2} C_{\sigma \tau}^{\beta} \rho^{\tau} \wedge \rho^{\sigma}\right) .
\end{aligned}
$$

Thus the image is isomorphic to $W(\mathfrak{g})$ and, with respect to the differential $d=\partial+(-1)^{q} \delta$, it is an acyclic subalgebra of $\Lambda(\eta \oplus \partial \eta \oplus \rho \oplus \partial \rho)$. The objective is to find a strong deformation retract of $\Lambda(\eta \oplus \partial \eta \oplus \rho \oplus \partial \rho)$ onto this subalgebra. To begin, let

$$
\varpi: \Lambda(\eta \oplus \partial \eta \oplus \rho \oplus \partial \rho) \rightarrow \Lambda(\eta \oplus \partial \eta \oplus \rho \oplus \partial \rho)
$$

be the algebra homomorphism defined on the generators by

$$
\begin{gathered}
\varpi\left(\eta^{\alpha}\right)=\eta^{\alpha}+\rho^{\alpha}, \quad \varpi\left(\partial \eta^{\alpha}\right)=\partial \rho^{\alpha}+\frac{1}{2} C_{\beta \gamma}^{\alpha} \rho^{\gamma} \wedge \rho^{\beta}, \\
\varpi\left(\rho^{\alpha}\right)=0, \quad \text { and } \quad \varpi\left(\partial \rho^{\alpha}\right)=\partial \rho^{\alpha}+\frac{1}{2} C_{\beta \gamma}^{\alpha} \rho^{\gamma} \wedge \rho^{\beta} .
\end{gathered}
$$

The image will be denoted by $\Lambda(\varpi(\eta) \oplus \varpi(\partial \eta))$ since $\varpi(\partial \eta)=\varpi(\partial \rho)$. Because the subalgebra $\Lambda(\eta \oplus \partial \eta)$ is isomorphic to $W(\mathfrak{g})$ and $\varpi$ restricted to this subalgebra is equivalent to the $\mathfrak{g}$-differential graded algebra homomorphism $(A, F): W(\mathfrak{g}) \rightarrow \Lambda(\eta \oplus \partial \eta \oplus \rho \oplus \partial \rho)$, it follows that $d \varpi\left(\eta^{\alpha}\right)=\varpi\left(d \eta^{\alpha}\right)$ and $d \varpi\left(\partial \eta^{\alpha}\right)=\varpi\left(d \partial \eta^{\alpha}\right)$. That $\varpi$ and $d$ commute on $\rho^{\alpha}$ and $\partial \rho^{\alpha}$ is shown by easy calculations. Thus $\varpi$ is a chain map from $\Lambda(\eta \oplus \partial \eta \oplus \rho \oplus \partial \rho)$ to itself. More easy calculations show that $\varpi$ commutes with the maps $i$ and $\theta$. Thus $\varpi$ is a $\mathfrak{g}$-differential graded algebra endomorphism of $\Lambda(\eta \oplus \partial \eta \oplus \rho \oplus \partial \rho)$.

To show that $\varpi$ is chain homotopic to the identity homomorphism requires a change of generators of $\Lambda(\eta \oplus \partial \eta \oplus \rho \oplus \partial \rho)$ so that a contracting homotopy $h$ can be defined easily. Note that the elements,

$$
\begin{gathered}
\eta^{\alpha}+\rho^{\alpha} \in \Lambda^{1}(\eta \oplus \partial \eta \oplus \rho \oplus \partial \rho), \\
\partial \rho^{\alpha}+\frac{1}{2} C_{\beta \gamma}^{\alpha} \rho^{\gamma} \wedge \rho^{\beta} \in \Lambda^{2}(\eta \oplus \partial \eta \oplus \rho \oplus \partial \rho), \\
\rho^{\alpha} \in \Lambda^{1}(\eta \oplus \partial \eta \oplus \rho \oplus \partial \rho),
\end{gathered}
$$

and

$$
\partial \rho^{\alpha}-\partial \eta^{\alpha}+C_{\beta \gamma}^{\alpha} \eta^{\gamma} \wedge \rho^{\beta} \in \Lambda^{2}(\eta \oplus \partial \eta \oplus \rho \oplus \partial \rho),
$$

are also a set of generators of $\Lambda(\eta \oplus \partial \eta \oplus \rho \oplus \partial \rho)$, where $\partial \rho^{\alpha}-\partial \eta^{\alpha}+C_{\beta \gamma}^{\alpha} \eta^{\gamma} \wedge \rho^{\beta}=$ $d\left(\rho^{\alpha}\right)$. With respect to these generators, the homomorphism $\varpi$ is defined by

$$
\begin{aligned}
\varpi\left(\eta^{\alpha}+\rho^{\alpha}\right) & =\eta^{\alpha}+\rho^{\alpha}, \\
\varpi\left(\partial \rho^{\alpha}+\frac{1}{2} C_{\beta \gamma}^{\alpha} \rho^{\gamma} \wedge \rho^{\beta}\right) & =\partial \rho^{\alpha}+\frac{1}{2} C_{\beta \gamma}^{\alpha} \rho^{\gamma} \wedge \rho^{\beta}, \\
\varpi\left(\rho^{\alpha}\right) & =0, \\
\varpi\left(\partial \rho^{\alpha}-\partial \eta^{\alpha}-C_{\beta \gamma}^{\alpha} \eta^{\gamma} \wedge \rho^{\beta}\right) & =0 .
\end{aligned}
$$

Thus, the image of $\varpi$, with respect to the generators, is still $\Lambda(\varpi(\eta) \oplus \varpi(\partial \eta))$. Define a vector space map $h$ by $h\left(\eta^{\alpha}+\rho^{\alpha}\right)=0, h\left(\partial \rho^{\alpha}+\frac{1}{2} C_{\beta \gamma}^{\alpha} \rho^{\gamma} \wedge \rho^{\beta}\right)=0$, $h\left(\rho^{\alpha}\right)=0$, and $h\left(\partial \rho^{\alpha}-\partial \eta^{\alpha}-C_{\beta \gamma}^{\alpha} \eta^{\gamma} \wedge \rho^{\beta}\right)=-\rho^{\alpha}$. 
Proposition 9.1. The map $h$ is a chain homotopy between $\varpi$ and the identity homomorphism Id.

Proof. That $h d+d h=\varpi-I$ on the generators is another easy calculation.

Lemma 9.2. Given a free differential graded algebra $A$, two degree zero differential graded algebra maps $f$ and $g$, if $h$ is a degree -1 graded vector space homomorphism such that $h d+d h=f-g$ on the generators of $A$, then $h$ can be extended to a chain homotopy on all of $A$.

Proof. Let $a, b$ be generators of $A$. By assumption it is known that $h d(a)+$ $d h(a)=f(a)-g(a)$ and similarly for $b$. The objective is to define $h(a b)$ so that

$$
h d(a b)+d h(a b)=f(a) f(b)-g(a) g(b) .
$$

Note that for $h$ to be well defined on the products of generators it must satisfy $l_{i}(a b)=(-1)^{\operatorname{deg}(a) \operatorname{deg}(b)} h(b a)$. The following definition of $h$ on a product satisfies these conditions

$$
\begin{aligned}
h(a b)= & \frac{1}{2}\left(h(a) f(b)+(-1)^{\operatorname{deg}(a)} g(a) h(b)\right) \\
& +\frac{1}{2}(-1)^{\operatorname{deg}(a) \operatorname{deg}(b)}\left(h(b) f(a)+(-1)^{\operatorname{deg}(b)} g(b) h(a)\right) .
\end{aligned}
$$

The proof that this definition does define $h$ for a product $a b$, and thus for all of $A$, reduces to a long algebraic computation.

Thus $h d+d h=\varpi$ - Id on the generators and it follows from Lemma 9.2 that the map $h$ extends to a chain homotopy and the proposition is proved.

Consider the $\mathfrak{g}$-differential graded algebra homomorphism

$$
\bar{\varpi}: \Lambda(\eta \oplus \partial \eta \oplus \rho \oplus \partial \rho) \rightarrow \Lambda(\varpi(\eta) \oplus \varpi(\partial \eta)),
$$

where $\bar{\varpi}$ is just $\varpi$ with its range restricted to its image, and the inclusion map

$$
i: \Lambda(\varpi(\eta) \oplus \varpi(\partial \eta)) \rightarrow \Lambda(\eta \oplus \partial \eta \oplus \rho \oplus \partial \rho) .
$$

Note that, on the one hand, $\varpi \circ i$ is the identity on $\Lambda(\varpi(\eta) \oplus \varpi(\partial \eta))$ and on the other hand, that $i \circ \bar{\varpi}=\varpi$.

Thus the $\mathfrak{g}$-differential graded algebra homomorphism,

$$
\bar{\varpi}: \Lambda(\eta \oplus \partial \eta \oplus \rho \oplus \partial \rho) \rightarrow \Lambda(\varpi(\eta) \oplus \varpi(\partial \eta)),
$$

is a strong deformation retraction of $\Lambda(\eta \oplus \partial \eta \oplus \rho \oplus \partial \rho)$ onto the subalgebra $\Lambda(\varpi(\eta) \oplus \varpi(\partial \eta))$ and hence induces an isomorphism in cohomology. (This should not be surprising since both the algebras are acyclic.)

Since $\eta^{\alpha}$ has form degree zero, powers of the generator $\eta^{\alpha}+\rho^{\alpha}$ will never be annihilated due to the dimension of the base space $M$. The generator $\partial \rho^{\alpha}+$ $\frac{1}{2} C_{\beta \gamma}^{\alpha} \rho^{\gamma} \wedge \rho^{\beta}$ has form degree two in both summands; it will be annihilated when it is raised to a power $t$, such that $2 t>n$. The generator $\rho^{\alpha}$ is annihilated when raised to a power $r$, with $r>n$. Finally, the generator $\partial \rho^{\alpha}-\partial \eta^{\alpha}+$ $C_{\beta \gamma}^{\alpha} \eta^{\gamma} \wedge \rho^{\beta}$ has inhomogeneous form degree, the first term has form degree two, while the last two terms have form degree one. It will be annihilated only if it is raised to a power $s$, such that $s>n$. Therefore, the ideal $I$, the kernel of the differential graded algebra homomorphism, $\Pi: \Lambda(\eta \oplus \partial \eta \oplus \rho \oplus \partial \rho) \rightarrow S_{U}^{\bullet}, \bullet$, with respect to these generators, is

$$
\left(\bigoplus_{2 r+s+l>n} S^{r}\left(\partial \rho^{\alpha}+\frac{1}{2} C_{\beta \gamma}^{\alpha} \rho^{\gamma} \wedge \rho^{\beta}\right) \otimes E^{s}\left(\rho^{\alpha}\right) \otimes S^{t}\left(\partial \rho^{\alpha}-\partial \eta^{\alpha}+C_{\beta \gamma}^{\alpha} \eta^{\gamma} \wedge \rho^{\beta}\right)\right) .
$$


The ideal $I$ is a differential ideal, since

$$
\begin{gathered}
d\left(\partial \rho^{\alpha}+\frac{1}{2} C_{\beta \gamma}^{\alpha} \rho^{\gamma} \wedge \rho^{\beta}\right)=-C_{\beta \gamma}^{\alpha}\left(\eta^{\gamma}+\rho^{\gamma}\right) \wedge\left(\partial \rho^{\beta}+\frac{1}{2} C_{\sigma \tau}^{\beta} \rho^{\tau} \wedge \rho^{\sigma}\right), \\
d\left(\rho^{\alpha}\right)=\partial \rho^{\alpha}-\partial \eta^{\alpha}+C_{\beta \gamma}^{\alpha} \eta^{\gamma} \wedge \rho^{\beta}
\end{gathered}
$$

and

$$
d\left(\partial \rho^{\alpha}-\partial \eta^{\alpha}+C_{\beta \gamma}^{\alpha} \eta^{\gamma} \wedge \rho^{\beta}\right)=0
$$

It follows that

$$
\begin{aligned}
& d\left(S^{r}\left(\partial \rho^{\alpha}+\frac{1}{2} C_{\beta \gamma}^{\alpha} \rho^{\gamma} \wedge \rho^{\beta}\right) \otimes E^{s}\left(\rho^{\alpha}\right) \otimes S^{t}\left(\partial \rho^{\alpha}-\partial \eta^{\alpha}+C_{\beta \gamma}^{\alpha} \eta^{\gamma} \wedge \rho^{\beta}\right)\right) \\
& \subset \bigoplus_{i=0}^{1}\left(S^{r+i}\left(\partial \rho^{\alpha}+\frac{1}{2} C_{\beta \gamma}^{\alpha} \rho^{\gamma} \wedge \rho^{\beta}\right)\right. \\
& \left.\otimes E^{s-1}\left(\rho^{\alpha}\right) \otimes S^{t+1}\left(\partial \rho^{\alpha}-\partial \eta^{\alpha}+C_{\beta \gamma}^{\alpha} \eta^{\gamma} \wedge \rho^{\beta}\right)\right)
\end{aligned}
$$

and thus $d(I) \subset I$, since if the weighted sum of first set of exponents is greater than $n$, then clearly so is the weighted sum of the second set of exponents. The chain homotopy $h$ sends $I$ to itself, since

$h\left(\partial \rho^{\alpha}+\frac{1}{2} C_{\beta \gamma}^{\alpha} \rho^{\gamma} \wedge \rho^{\beta}\right)=0, \quad h\left(\rho^{\alpha}\right)=0, \quad h\left(\partial \rho^{\alpha}-\partial \eta^{\alpha}+C_{\beta \gamma}^{\alpha} \eta^{\gamma} \wedge \rho^{\beta}\right)=\rho^{\alpha}$, and a computation shows that

$$
\begin{aligned}
& h\left(S^{r}\left(\partial \rho^{\alpha}+\frac{1}{2} C_{\beta \gamma}^{\alpha} \rho^{\gamma} \wedge \rho^{\beta}\right) \otimes E^{s}\left(\rho^{\alpha}\right) \otimes S^{t}\left(\partial \rho^{\alpha}-\partial \eta^{\alpha}+C_{\beta \gamma}^{\alpha} \eta^{\gamma} \wedge \rho^{\beta}\right)\right) \\
& \quad \subset S^{r}\left(\partial \rho^{\alpha}+\frac{1}{2} C_{\beta \gamma}^{\alpha} \rho^{\gamma} \wedge \rho^{\beta}\right) \otimes E^{s+1}\left(\rho^{\alpha}\right) \otimes S^{t-1}\left(\partial \rho^{\alpha}-\partial \eta^{\alpha}+C_{\beta \gamma}^{\alpha} \eta^{\gamma} \wedge \rho^{\beta}\right) .
\end{aligned}
$$

Consequently, it follows that $h(I) \subset I$. The homomorphism $\varpi$ sends the ideal $I$. onto the ideal $I^{\prime}$,

$$
\left(\bigoplus_{2 r>n} S^{r}\left(\partial \rho^{\alpha}+\frac{1}{2} C_{\beta \gamma}^{\alpha} \rho^{\gamma} \wedge \rho^{\beta}\right)\right)=\left(\bigoplus_{2 r>n} S^{r}(\varpi(\partial \eta))\right),
$$

which is also a differential ideal, since as was shown above

$$
d\left(\partial \rho^{\alpha}+\frac{1}{2} C_{\beta \gamma}^{\alpha} \rho^{\gamma} \wedge \rho^{\beta}\right)=-C_{\beta \gamma}^{\alpha}\left(\eta^{\gamma}+\rho^{\gamma}\right) \wedge\left(\partial \rho^{\beta}+\frac{1}{2} C_{\sigma \tau}^{\beta} \rho^{\tau} \wedge \rho^{\sigma}\right) .
$$

A straightforward calculation shows that on the generators of $I, h d+d h=\varpi-$ Id. Hence $\bar{\varpi}: I \rightarrow I^{\prime}$ will produce an isomorphism between the cohomology of $I$ and the cohomology of $I^{\prime}$. Finally, it follows that the diagram

0

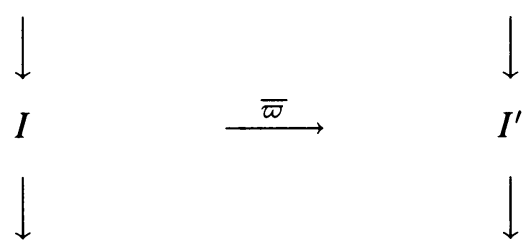

$\Lambda(\eta \oplus \partial \eta \oplus \rho \oplus \partial \rho) \stackrel{\bar{\varpi}}{\longrightarrow} \Lambda(\varpi(\eta) \oplus \varpi(\partial \eta))$

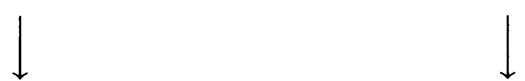

$\Lambda(\eta \oplus \partial \eta \oplus \rho \oplus \partial \rho)_{q \leq n} \stackrel{\check{\varpi}}{\longrightarrow} \Lambda(\varpi(\eta) \oplus \varpi(\partial \eta)) / I^{\prime}$ 
is a commutative diagram of $\mathfrak{g}$-differential graded algebras and $\bar{\varpi}$ induces an isomorphism in cohomology in the first two rows. Thus $\tilde{\varpi}$ induces an isomorphism in cohomology in the last row. As was shown above, the algebra $\Lambda(\varpi(\eta) \oplus \varpi(\partial \eta))$, with the differential $d$, is isomorphic to $W(\mathfrak{g})$, but the ideal $I^{\prime}$ is isomorphic to the ideal $\left(\bigoplus_{2 r>n} S^{r}\left(\sigma \mathfrak{g}^{*}\right)\right)$ in $W(\mathfrak{g})$. Consequently there is an isomorphism

$$
\Lambda(\varpi(\eta) \oplus \varpi(\partial \eta)) / I^{\prime} \simeq W(\mathfrak{g}) /\left(\bigoplus_{2 r>n} S^{r}\left(\sigma \mathfrak{g}^{*}\right)\right) \simeq W(\mathfrak{g})_{\left[\frac{n}{2}\right]}
$$

It follows that for any compact, connected Lie group $G$ with Lie algebra $\mathfrak{g}$,

$$
H_{d}^{\bullet}\left(\left(S_{U}^{\bullet, \bullet}\right)^{\operatorname{Diff}_{\mathrm{loc}}\left(\mathbb{R}^{n}\right)}\right) \simeq H_{d}^{\bullet}\left(W(\mathfrak{g})_{\left[\frac{n}{2}\right]}\right) .
$$

This proves the theorem.

\section{A THEOREM ON GLOBALIZATION}

Theorem 10. Let $P$ be a principal fiber bundle. If $\operatorname{ad}^{*} P$ is trivializable, where $\operatorname{ad}^{*} P$ is the vector bundle with fiber $\mathfrak{g}^{*}$ associated to $P$ via the $\mathrm{Ad}^{*}$ representation of $G$ in $\operatorname{Aut}\left(\mathfrak{g}^{*}\right)$, then there exists a $\mathfrak{g}$-differential graded algebra, denoted by

$$
\left(\Lambda(H \oplus \partial H \oplus R \oplus \partial R)_{q \leq n}, d\right)
$$

of globally defined differential operators with the following properties:

$$
\left(\Lambda(H \oplus \partial H \oplus R \oplus \partial R)_{q \leq n}, d\right) \simeq\left(\Lambda(\eta \oplus \partial \eta \oplus \rho \oplus \partial \rho)_{q \leq n}, d\right)
$$

where the two algebras are isomorphic as $\mathfrak{g}$-differential graded algebras; thus the cohomology of $(\Lambda(H \oplus \partial H \oplus R \oplus \partial R), d)$ is isomorphic to $H_{d}^{\bullet}\left(W(\mathfrak{g})_{\left[\frac{n}{2}\right]}\right)$, and

$$
\left.\Lambda(H \oplus \partial H \oplus R \oplus \partial R)_{q \leq n}\right|_{U} \simeq\left(S_{U}^{\bullet, \bullet}\right)^{\operatorname{Diff}_{\mathrm{loc}}\left(\mathbb{R}^{n}\right)}
$$

over open sets $U$ of $M$.

Proof. By definition $\operatorname{ad}^{*} P$ is the set of the equivalence classes in $P \times \mathfrak{g}^{*}$ modulo the action of $G$, where the representation of $G$ in $\operatorname{Aut}\left(\mathfrak{g}^{*}\right)$ is given by the co-adjoint action of $G$ on $\mathfrak{g}^{*}$. Thus the equivalence class of the point $(p, \eta)$ is the set $\left\{\left(p g, \operatorname{Ad}^{*}\left(g^{-1}\right) \eta\right) \mid g \in G\right\}$. Note, on the one hand, that there is the canonical pairing $\left\langle\Gamma\left(\operatorname{ad}^{*} P\right), \Gamma(\operatorname{ad} P)\right\rangle \rightarrow C^{\infty}(P)^{G}$, where $\Gamma(\operatorname{ad} P) \simeq$ $\Omega^{0}\left(M\right.$, ad $P$ ) and $C^{\infty}(P)^{G}$ is the algebra of $G$-invariant functions on $P$. But if a function on $P$ is $G$-invariant, it drops to a function on the base space $M$. Thus, the above pairing produces a smooth function on $M$. On the other hand, there also is the canonical pairing $\left\langle\Gamma\left(\operatorname{ad}^{*} P\right), \Omega^{1}(M\right.$, ad $\left.P)\right\rangle \rightarrow$ $\Omega^{1}\left(M, C^{\infty}(P)^{G}\right)$ which is defined on sections $\omega \otimes s$ of $\Omega^{1}(M$, ad $P)$ by $\langle\sigma, \omega \otimes s\rangle=\omega \sigma(s)$, where $\sigma \in \Gamma\left(\operatorname{ad}^{*} P\right), \omega \in \Omega^{1}(M)$, and $s \in \Gamma(\operatorname{ad} P)$. Note that $\Omega^{1}\left(M, C^{\infty}(P)^{G}\right)$ is isomorphic to $\Omega^{1}(M)$. In general $\Gamma\left(\operatorname{ad}^{*} P\right)$ is $\Omega^{0}\left(M, \operatorname{ad}^{*} P\right)$ in analogy to the situation for $\Gamma(\operatorname{ad} P)$. Note that $\Omega^{0}\left(M, \operatorname{ad}^{*} P\right)$ is the zero graded vector space of the graded algebra $\Omega^{\bullet}\left(M, \operatorname{ad}^{*} P\right)$. Furthermore given a covariant derivative $D$ for $\Omega^{\bullet}\left(M, \operatorname{ad}^{*} P\right)$, there is a connection $A$ on $P$ such that the induced covariant derivative $d_{A}^{*}$ is equivalent to the covariant derivative $D$ (the superscript ${ }^{*}$ is included to distinguish the induced covariant derivative on $\operatorname{ad}^{*} P$ from the induced covariant derivative on ad $P$ which has been denoted by $d_{A}$ ). 
Note that $\operatorname{ad}^{*} P$ being trivializable means that $\operatorname{ad}^{*} P$ is equivalent to $M \times$ $\mathfrak{g}^{*}$ and thus there is an injective vector space homomorphism of $\mathfrak{g}^{*}$ into the sections of $M \times \mathfrak{g}^{*}$. The image may be taken to be the global constant sections. Furthermore, $\operatorname{ad}^{*} P$ being trivializable implies that $\Omega^{\bullet}\left(M, \operatorname{ad}^{*} P\right)$ admits $d_{M}$, the exterior derivative of $M$, as a covariant derivative. Thus, as was noted above, there is a connection $A_{0}$ on $P$ such that the induced covariant derivative $d_{A_{0}}^{*}$ is just $d_{M}$. Furthermore, note that if $i\left(\eta^{\alpha}\right)$ denotes the image of a basis element of $\mathfrak{g}^{*}$ in $\Omega^{0}\left(M, \mathrm{ad}^{*} P\right)$, then $d_{M}\left(i\left(\eta^{\alpha}\right)\right)=d_{A_{0}}^{*}\left(i\left(\eta^{\alpha}\right)\right)=0$. It is from this image of $\mathfrak{g}^{*}$ in $\Gamma\left(\operatorname{ad}^{*} P\right)$, the global constant sections, and the connection $A_{0}$ that the $\mathfrak{g}$-differential graded algebra $(\Lambda(H \oplus \partial H \oplus R \oplus \partial R), d)$ will be constructed.

Since there is an injective map of $\mathfrak{g}^{*}$ into $\Omega^{0}\left(M, \operatorname{ad}^{*} P\right)$, then, via the two pairings described above, there is a pairing of $\mathfrak{g}^{*}$ with both $\Omega^{0}(M$, ad $P)$ and $\Omega^{1}(M$, ad $P)$. To distinguish between these two pairings, the image of $\mathfrak{g}^{*}$ in $\Omega^{0}\left(M, \operatorname{ad}^{*} P\right)$ will be denoted by two different letters. When $i\left(\mathfrak{g}^{*}\right)$ is to be paired with $\Gamma($ ad $P)$, it will be denoted by $H$ and basis elements of $H$ by $H^{\alpha}$, where $\alpha$ is a Lie algebra index, whereas when $i\left(\mathfrak{g}^{*}\right)$ is to be paired with $\Omega^{1}(M$, ad $P)$ then it will be denoted by $R$ and basis elements of $R$ by $R^{\alpha}$ ( $H$ and $R$ should be thought of as capital latin versions of $\eta$ and $\rho$ ).

Note that, on the one hand, there are the following relationships between $H$, $\eta, R$, and $\rho$

$$
H: \Gamma(\operatorname{ad} P) \rightarrow \Omega^{0}(M), \quad \text { while } \eta:\left.\left.\Gamma(\operatorname{ad} P)\right|_{U} \rightarrow \Omega^{0}(M)\right|_{U},
$$

and

(2) $\quad R: \Omega^{1}(M$, ad $P) \rightarrow \Omega^{1}(M), \quad$ while $\rho:\left.\left.\Omega^{1}(M$, ad $P)\right|_{U} \rightarrow \Omega^{1}(M)\right|_{U}$.

On the other hand, notice that the vector spaces of operators $\partial \eta$ and $\partial \rho$ have the following domains and ranges:

$$
\partial \eta:\left.\left.\Gamma(\operatorname{ad} P)\right|_{U} \rightarrow \Omega^{1}(M)\right|_{U}, \quad \text { and } \partial \rho:\left.\left.\Omega^{1}(M, \text { ad } P)\right|_{U} \rightarrow \Omega^{2}(M)\right|_{U},
$$

where $\partial \eta$ is given by the composition

$$
\left.\left.\left.\Gamma(\operatorname{ad} P)\right|_{U} \stackrel{\eta}{\longrightarrow} \Omega^{0}(M)\right|_{U} \stackrel{d_{M}}{\longrightarrow} \Omega^{1}(M)\right|_{U}
$$

and $\partial \rho$ is given by the composition

$$
\left.\left.\left.\Omega^{1}(M, \text { ad } P)\right|_{U} \stackrel{\rho}{\longrightarrow} \Omega^{1}(M)\right|_{U} \stackrel{d_{M}}{\longrightarrow} \Omega^{2}(M)\right|_{U} .
$$

Thus $\partial H$ and $\partial R$ will be defined similarly as $\partial H \equiv d_{M} \circ H$ and $\partial R \equiv d_{M} \circ R$, that is $\partial H: \Gamma(\operatorname{ad} P) \rightarrow \Omega^{1}(M)$ is given by the composition

$$
\Gamma(\operatorname{ad} P) \stackrel{H}{\longrightarrow} \Omega^{0}(M) \stackrel{d_{M}}{\longrightarrow} \Omega^{1}(M)
$$

and $\partial R: \Omega^{1}(M$, ad $P) \rightarrow \Omega^{2}(M)$ is given by the composition

$$
\Omega^{1}(M, \text { ad } P) \stackrel{R}{\longrightarrow} \Omega^{1}(M) \stackrel{d_{M}}{\longrightarrow} \Omega^{2}(M) .
$$

Clearly, since the elements of $H$ and $R$ are nowhere vanishing sections of $\operatorname{ad}^{*} P$, as operators they decrease supports; furthermore since the exterior derivative $d$ on $M$ is a local operator the operators in $\partial H$ and $\partial R$ also 
decrease supports. Thus by Peetre's Theorem all four types of operators are differential operators. The first two are differential operators of order zero while the latter two are differential operators of order one.

Consider the graded algebra $\Lambda(H \oplus \partial H \oplus R \oplus \partial R)$ where $H$ has total degree one and bidegree $(1,0), \partial H$ has total degree two and bidegree $(1,1)$, $R$ has total degree one and bidegree $(0,1)$, and $\partial R$ has total degree two and bidegree $(0,2)$ (these are the degrees and bidegrees of $\eta, \partial \eta, \rho$, and $\partial \rho$ respectively and as before the first bidegree represents the number of arguments from $\Omega^{0}(M$, ad $P)$ and the second bidegree represents the degree of the form produced by the operator). As a graded algebra, it is isomorphic to $\Lambda(\eta \oplus \partial \eta \oplus \rho \oplus \partial \rho)$, the isomorphism being the obvious one, namely $H^{\alpha}$ is sent to $\eta^{\alpha}$ and similarly for the other generators. Note that a typical basis element in $\Lambda(H \oplus \partial H \oplus R \oplus \partial R)$ is of the form

$$
H^{\alpha_{1}} \wedge \cdots \wedge H^{\alpha_{i}} \wedge \partial H^{\alpha_{i+1}} \wedge \cdots \wedge \partial H^{\alpha_{p}} \wedge R^{\beta_{1}} \wedge \cdots \wedge R^{\beta_{j}} \wedge \partial R^{\beta_{j+1}} \wedge \cdots \wedge \partial R^{\beta_{m}}
$$

where as before the wedge $\wedge$ denotes the graded product. Furthermore the above basis element is a globally defined differential operator from $p$ copies of $\Omega^{0}(M$, ad $P)$ and $m$ copies of $\Omega^{1}(M$, ad $P)$ to $q$-forms of $M$ where $q=(p-i)+j+2(m-j)$. As before if $q>n$, where $n$ is the dimension of $M$, a product of the above type is zero, since the form degree exceeds the dimension of the manifold $M$. The ideal $I^{\prime}$ of operators whose form degree $q$ is greater than $n$ is isomorphic to the ideal $I$ described in $\S 9$. It follows that there is a commutative diagram

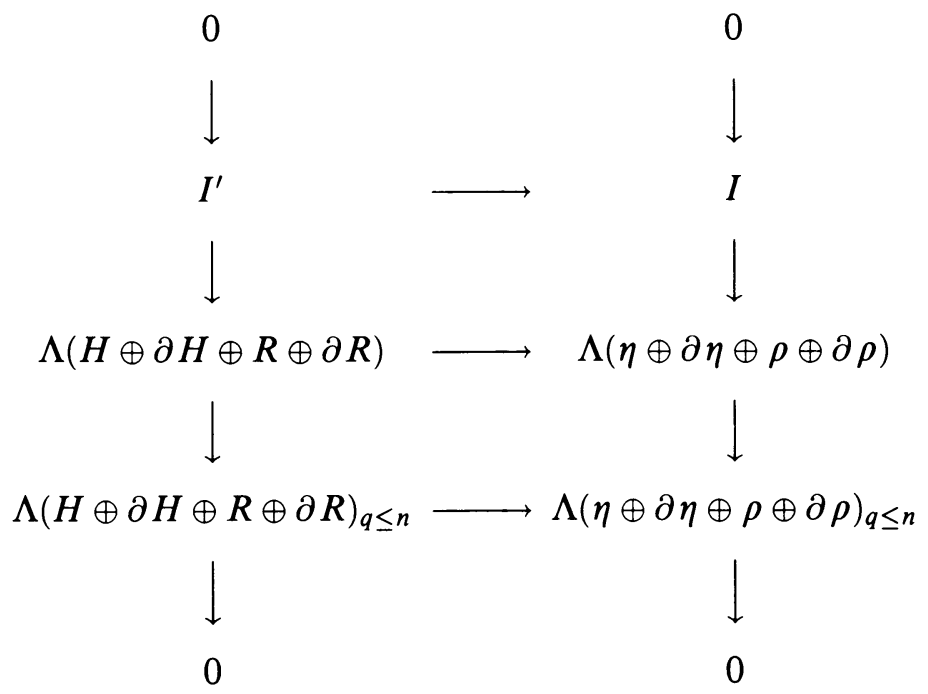

where $\Lambda(H \oplus \partial H \oplus R \oplus \partial R)_{q \leq n}$ denotes the quotient, the first horizontal map is an isomorphism of graded ideals, and the last two horizontal maps are isomorphisms of graded algebras.

The two differentials $\partial$ and $\delta$ are well defined for the graded algebra of global differentials operators. Specifically they are defined by the same formulas as before, i.e. if $L \in \Lambda(H \oplus \partial H \oplus R \oplus \partial R)$ is of the form above, $f_{1}, \ldots f_{j} \in \Omega^{0}(M$, ad $P)$, and $\omega \in \Omega^{1}(M$, ad $P)$ then $(\partial L)\left(f_{1}, \ldots, f_{j}, \omega\right)=$ 
$d\left(L\left(f_{1}, \ldots, f_{j}, \omega\right)\right.$ while

$$
\begin{gathered}
(\delta L)\left(f_{1}, \ldots, f_{j+1}, \omega\right)=\sum_{i}(-1)^{i} L\left(f_{1}, \ldots, \hat{f}_{i}, \ldots, f_{j+1}, d_{A_{0}} f_{i}+[\omega, f]\right) \\
\quad+\sum_{i<j}(-1)^{i+j} L\left(\left[f_{i}, f_{j}\right], f_{1}, \ldots, \hat{f}_{i}, \ldots, \hat{f}_{j}, \ldots, f_{j+1}, \omega\right)
\end{gathered}
$$

where the covariant derivative $d_{A_{0}}$ comes from the connection $A_{0}$ described above and, as always, Chern's convention is being used for the argument $\omega$.

Clearly the differential $\partial$ is exactly the same for $\Lambda(H \oplus \partial H \oplus R \oplus \partial R)$ as it was for $\Lambda(\eta \oplus \partial \eta \oplus \rho \oplus \partial \rho)$ since on the generators $\partial\left(H^{\alpha}\right)=\partial H^{\alpha}$ and $\partial\left(R^{\alpha}\right)=\partial R^{\alpha}$. The only potential problem is the decomposition of $\delta$ into the two separate differentials $\vartheta$, the "coadjoint action" of elements of $\Gamma(\operatorname{ad} P)$, and the twisting differential $\tau$. Recall that $\tau\left(\rho^{\alpha}\right)=\partial \eta^{\alpha}$ because over an open set $U$ of $M$ it is possible to choose the background connection $A_{0}$ so that its induced covariant connection is the exterior derivative, and thus $\partial \eta^{\alpha}(f)=d_{\mathbb{R}^{n}} f^{\alpha}=$ $\rho^{\alpha}\left(d_{\mathbb{R}^{n}} f\right)=\rho^{\alpha}\left(d_{A_{0}} f\right)$. The analogous property in this setting is that there is a twisting differential, also denoted $\tau$, such that for all $R^{\alpha} \in R, \tau\left(R^{\alpha}\right)=\partial H^{\alpha}$ or equivalently $\partial H^{\alpha}(f)=R^{\alpha}\left(d_{A_{0}} f\right)$ for all $f \in \Gamma(\operatorname{ad} P)$. This is where the choice of $A_{0}$ made above becomes crucial.

Note that just as $\Omega^{0}\left(M\right.$, ad $\left.^{*} P\right)$ can be paired with $\Omega^{1}(M$, ad $P)$ to produce a one-form on $M$, the vector space $\Omega^{1}\left(M, \operatorname{ad}^{*} P\right)$ can be paired with $\Omega^{0}(M$, ad $P)$ to produce a one-form on $M$. It follows in general [10] that for a connection $A$ on $P, \sigma \in \Omega^{0}\left(M, \operatorname{ad}^{*} P\right)$ and $f \in \Omega^{0}(M$, ad $P)$, there is the equality $d_{M}\langle\sigma, f\rangle=\left\langle d_{A}^{*} \sigma, f\right\rangle+\left\langle\sigma, d_{A} s\right\rangle$, where

$$
d_{A}^{*}: \Omega^{0}\left(M, \operatorname{ad}^{*} P\right) \rightarrow \Omega^{1}\left(M, \operatorname{ad}^{*} P\right)
$$

and

$$
d_{A}: \Omega^{0}(M, \text { ad } P) \rightarrow \Omega^{1}(M, \text { ad } P)
$$

are the induced covariant derivatives on $\Omega^{\bullet}\left(M, \operatorname{ad}^{*} P\right)$ and $\Omega^{\bullet}(M$, ad $P)$ respectively. The pairing $\langle$,$\rangle must be interpreted according to its arguments,$ i.e. on the left-hand side it is the pairing of $\Omega^{0}\left(M, \operatorname{ad}^{*} P\right)$ with $\Omega^{0}(M$, ad $P)$ and so on. In the present setting only the subspace $H$ is being paired with elements of $\Gamma(\operatorname{ad} P)$. Thus the above equation becomes

$$
d_{M}\left\langle H^{\alpha}, f\right\rangle=\left\langle d_{A_{0}}^{*} H^{\alpha}, f\right\rangle+\left\langle H^{\alpha}, d_{A_{0}} f\right\rangle,
$$

for each $H^{\alpha} \in H$. Note that on the one hand, $d_{M}\left\langle H^{\alpha}, f\right\rangle$ is by definition $\partial H^{\alpha}(f)$, while $\left\langle d_{A_{0}}^{*} H^{\alpha}, f\right\rangle=0$ since by the choice of the background connection $A_{0}, d_{A_{0}}^{*} H^{\alpha}=0$. On the other hand, $\left\langle H^{\alpha}, d_{A_{0}} f\right\rangle=R^{\alpha}\left(d_{A_{0}} f\right)$, because the same element of $\Omega^{0}\left(M, \operatorname{ad}^{*} P\right)$ is being paired with $d_{A_{0}} f$, an element of $\Omega^{1}(M$, ad $P)$. Hence it follows that $\partial H^{\alpha}(f)=d_{M}\left\langle H^{\alpha}, f\right\rangle=R^{\alpha}\left(d_{A_{0}} f\right)$. Thus on the generators $R^{\alpha}$ it follows that the differential $\delta$ decomposes into $\tau$ and $\vartheta$ since

$$
\begin{aligned}
\left(\delta R^{\alpha}\right)(f, \omega) & =R^{\alpha}\left(d_{A_{0}} f+[f, \omega]\right)=R^{\alpha}\left(d_{A_{0}} f\right)+R^{\alpha}([f, \omega]) \\
& =\partial H^{\alpha}(f)+\left(\vartheta(f) R^{\alpha}\right)(\omega)=\left(\tau\left(R^{\alpha}\right)\right)(f)+\left(\vartheta(f) R^{\alpha}\right)(\omega)
\end{aligned}
$$

where $\tau$ is defined by $\tau\left(R^{\alpha}\right)=\partial H^{\alpha}$ and $\vartheta$ is defined as in $\S 8$. What is left to check is that $\delta$ is just $\vartheta$ on the generators $\partial R^{\alpha}$, i.e. $\tau\left(\partial R^{\alpha}\right)=0$. This follows from the fact that $\delta$ and $\partial$ commute and the following simple calculation: 


$$
\delta\left(\partial R^{\alpha}\right)=\partial\left(\delta R^{\alpha}\right)=\partial\left(\tau\left(R^{\alpha}\right)+\vartheta\left(R^{\alpha}\right)\right)=\partial\left(\partial H^{\alpha}\right)+\partial\left(\vartheta\left(R^{\alpha}\right)\right)=\vartheta\left(\partial R^{\alpha}\right) .
$$

Thus with the differential $d=\partial+(-1)^{q} \delta$, where as before $q$ is the form degree, the above diagram is in fact a commuting diagram of differential graded ideals and differential graded algebras, and the isomorphisms are isomorphisms of differential graded ideals and differential graded algebras. The $\mathfrak{g}$ structure is defined by the action of $\mathfrak{g}$ on $\mathfrak{g}^{*}$, translated to the image of $\mathfrak{g}^{*}$ in $\Gamma\left(\operatorname{ad}^{*} P\right)$, and thus to the generators of $\Lambda(H \oplus \partial H \oplus R \oplus \partial R)$, i.e. $\operatorname{ad}^{*}\left(\eta_{\alpha}\right)\left(H^{\beta}\right)=$ $i\left(\operatorname{ad}^{*}\left(\eta_{\alpha}\right)\left(\eta^{\beta}\right)\right)$. It follows that $\Lambda(H \oplus \partial H \oplus R \oplus \partial R)$ and $\Lambda(\eta \oplus \partial \eta \oplus \rho \oplus \partial \rho)$ are isomorphic as $\mathfrak{g}$-differential algebras and thus the cohomology of $\Lambda(H \oplus \partial H \oplus R \oplus \partial R)_{q \leq n}$ is isomorphic to $H_{d}^{\bullet}\left(W(\mathfrak{g})_{\left[\frac{n}{2}\right]}\right)$.

The last part of the theorem that $\left.\Lambda(H \oplus \partial H \oplus R \oplus \partial R)\right|_{U} \simeq\left(S_{U}^{\bullet, \bullet}\right)^{\operatorname{Diff}_{\text {loc }}\left(\mathbf{R}^{n}\right)}$ follows from the simple observation that since the generators of $\Lambda(H \oplus \partial H \oplus R \oplus \partial R)$ are global constant sections, their restrictions to any open subset $U$ of $M$ are also constant. Thus, for example, $\left.H^{\alpha}\right|_{U}$ is exactly $\eta^{\alpha} \in$ $\left(S_{U}^{\bullet} \bullet\right)^{\text {Diffloc }}\left(\mathbb{R}^{n}\right)$.

Finally, there are the following two corollaries whose proofs are obvious and therefore are omitted.

Corollary 1. If $P$ is trivializable, then the $\mathfrak{g}$-differential graded algebra $(\Lambda(H \oplus \partial H \oplus R \oplus \partial R), d)$ of global differential operators exists.

Corollary 2. If the structure group $G$ of $P$ is abelian, then the $\mathfrak{g}$-differential graded algebra $(\Lambda(H \oplus \partial H \oplus R \oplus \partial R), d)$ of global differential operators exists.

\section{FUTURE DIRECTIONS}

In general $\operatorname{ad}^{*} P$ is not trivializable and thus the above theorem will not be applicable to all principal fiber bundles $P$. Thus a globalization for arbitrary principal fiber bundles $P$ will have to accomplished by some other method. A promising approach is to examine the g-invariant elements of $\Lambda(\eta \oplus \partial \eta \oplus \rho \oplus \partial \rho)_{q \leq n}$. Since the homomorphism $\varpi$ of Chapter 10 is a $\mathfrak{g}$-differential graded algebra homomorphism, there is the commuting diagram of the local structures

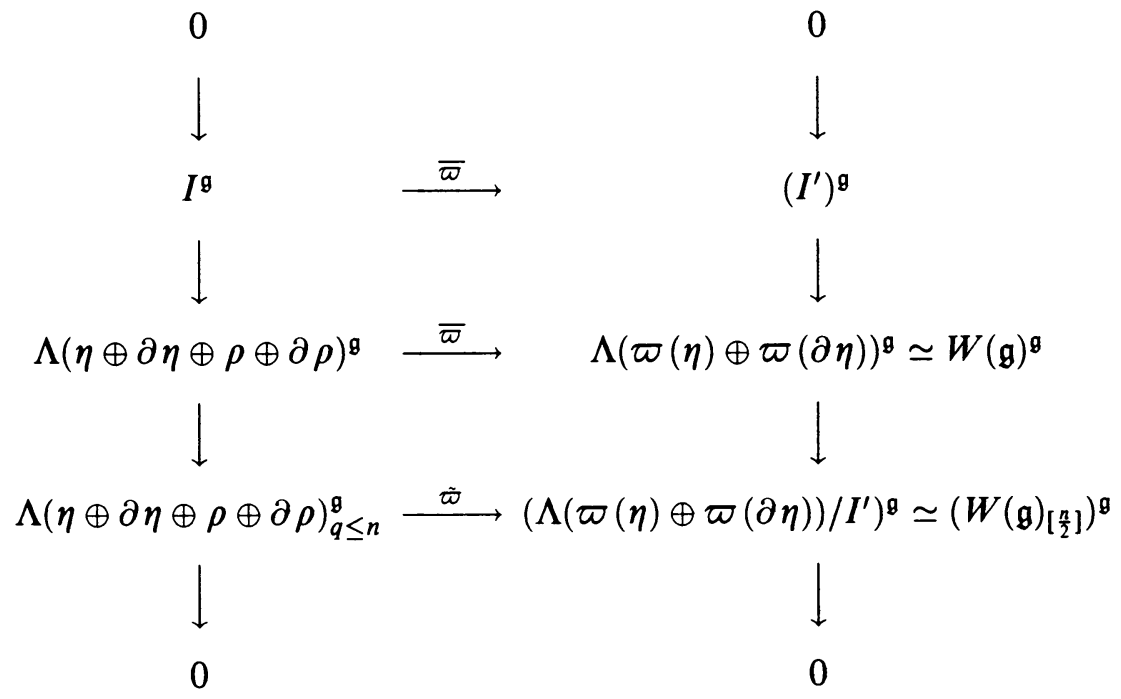


It is well known [7] that

$$
H_{d}^{\bullet}\left(\left(W(\mathfrak{g})_{\left[\frac{n}{2}\right]}\right)^{\mathfrak{g}}\right) \simeq H_{d}^{\bullet}\left(W(\mathfrak{g})_{\left[\frac{n}{2}\right]}\right)
$$

and it is hoped that the differential operators described by

$$
\Lambda(\eta \oplus \partial \eta \oplus \rho \oplus \partial \rho)_{q \leq n}^{\mathfrak{g}}
$$

will patch to global differential operators for nontrivial principal fiber bundles. If this is the case, then the result of the above theorem would globalize to the nontrivial, nonabelian case, i.e. there would be a $\mathfrak{g}$-differential graded algebra of globally defined differential operators whose cohomology would still be isomorphic to the cohomology of the Weil algebra truncated at $\left[\frac{n}{2}\right]$.

\section{REFERENCES}

1. M. F. Atiyah and R. Bott, The Yang-Mills equations over Riemann surfaces, Philos. Trans. Roy: Soc. London A 308 (1982), 523-615.

2. M. F. Atiyah and I. M. Singer, Dirac operators coupled to vector potentials, Proc. Nat. Acad. Sci. U.S.A. 81 (1984), 2597-2600.

3. L. Bonora and P. Cotta-Ramusino, Some remarks on B. R. S. transformations, anomalies and the cohomology of the Lie algebra of the group of gauge transformations, Comm. Math. Phys. 87 (1983), 589-603.

4. H. Cartan, Colloque de topologie (espaces fibres), tenu a Bruxelles du 5 au 8 Juin 1950, Centre Belge de Recherches Mathematiques, Thone, 1951.

5. P. Cotta-Ramusino, Anomalies and their cancelation, Fundamental aspects of quantum theory (V. Gorini and A. Frigerio, eds.), Plenum Press, New York and London, 1986, pp. 411-417.

6. P. Cotta-Ramusino and C. Reina, The action of the group of bundle-automorphisms on the space of connections and the geometry of gauge theories, J. Geom. Phys. 1 (1984), 121-155.

7. M. De Wilde and P. Lecompte, Cohomology of the Lie algebra of a manifold associated to the Lie derivative of smooth forms, J. Math. Pures Appl. 62 (1983), 197.

8. D. B. Fuks, Cohomology infinite-dimensional Lie algebras, Consultants Bureau, New York, 1986.

9. W. Greub, S. Halperin, and R. Van Stone, Connections, curvature, and cohomology, Academic Press, 1976.

10. C. Godbillon, Cohomologies d'algèbres de Lie de champs de vecteurs formels, Séminaire Bourbaki, vol. 1972/73, Exposés 418-435, Lecture Notes in Math., vol. 383, SpringerVerlag, 1974.

11. S. Halperin et D. Lehmann, Cohomologies et classes caracteristiques des choux de bruxelles, Univ. of Toronto, preprint.

12. A. Haefliger, Sur la cohomologie de l'algébre de Lie des champs de vecteurs, Ann. Sci. École Norm. Sup 4 (1976), 503-532.

13. J. L. Koszul, Homologie et cohomologie des algèbres de Lie, Bull. Soc. Math. France 78 (1950), 65.

14. P. K. Mitter and C. M. Vialette, On the bundle of connections and the gauge orbit manifold in Yang-Mills theory, Comm. Math. Phys. 79 (1981), 457.

15. M. S. Narasimhan and T. R. Ramadas, Geometry of $S U(\tau)$ gauge fields, Comm. Math. Phys. 67 (1979), 121.

16. J. Peetre, Une caracterisation abstraite des opérateurs différentiels, Math. Scand. 7 (1959), 211-218.

17. R. Schmidt, Local cohomology in gauge theories, Emory Univ., preprint.

18. M. Spivak, A comprehensive introduction to differential geometry, 2nd ed., Publish or Perish, 1979. 
19. T. Tsujishita, Continuous cohomology of the Lie algebra of vector fields, Mem. Amer. Math. Soc., vol. 34, no. 253, 1981.

20. H. Weyl, Classical groups, Princeton Univ. Press, 1939.

Department of Mathematics, East Carolina University, Greenville, North Carolina 27858

E-mail address, MAPETERS@ECUVML.BITNET

E-mail address: PETERSON@WORLD.STD.cOM 